Article

\title{
Facile and Efficient Syntheses of a Series of $N$-Benzyl and $N$-Biphenylmethyl Substituted Imidazole Derivatives Based on (E)-Urocanic acid, as Angiotensin II AT1 Receptor Blockers
}

\author{
George Agelis ${ }^{1,2, *}$, Konstantinos Kelaidonis ${ }^{1,2}$, Amalia Resvani ${ }^{1,2}$, Dimitra Kalavrizioti ${ }^{3}$, \\ Maria-Eleni Androutsou ${ }^{1,2}$, Panagiotis Plotas ${ }^{2,3}$, Demetrios Vlahakos ${ }^{4}$, \\ Catherine Koukoulitsa ${ }^{5}$, Theodore Tselios ${ }^{1}$, Thomas Mavromoustakos ${ }^{5}$ and \\ John Matsoukas ${ }^{1,2, *}$ \\ 1 Department of Chemistry, University of Patras, Patras 26500, Greece \\ 2 Eldrug S.A., Patras Science Park, Patras 26504, Greece \\ 3 Department of Pharmacology, School of Medicine, University of Patras, Patras 26500, Greece \\ 4 Department of Internal Medicine, 'ATTIKON' University Hospital, Athens 12462, Greece \\ 5 Department of Chemistry, University of Athens, Athens 15771, Greece \\ * Authors to whom correspondence should be addressed: \\ E-Mails: aggelisgeorge@hotmail.com (G.A.); imats@upatras.gr (J.M.)
}

Received: 24 April 2013; in revised form: 17 June 2013 / Accepted: 20 June 2013 /

Published: 27 June 2013

\begin{abstract}
In the present work, a facile and efficient route for the synthesis of a series of $N$-substituted imidazole derivatives is described. Docking studies have revealed that $N$-substituted imidazole derivatives based on $(E)$-urocanic acid may be potential antihypertensive leads. Therefore, new AT1 receptor blockers bearing either the benzyl or the biphenylmethyl moiety at the $N-1$ or $N-3$ position, either the $(E)$-acrylate or the propanoate fragment and their related acids at the $C-4$ position as well as a halogen atom at the $C-5$ position of the imidazole ring, were synthesized. The newly synthesized analogues were evaluated for binding to human AT1 receptor. The biological results showed that this class of molecules possesses moderate or no activity, thus not always confirming high docking scores. Nonetheless, important conclusions can be derived for their molecular basis of their mode of action and help medicinal chemists to design and synthesize more potent ones. An aliphatic group as in losartan seems to be important for enhancing binding affinity and activity.
\end{abstract}


Keywords: synthesis; AT1 receptor blockers; (E)-urocanic acid; $N$-alkylation; docking studies

\section{Introduction}

Angiotensin II (ANG II) is the octapeptide produced by the Renin-Angiotensin System (RAS) which plays a key role in the pathophysiology of hypertension [1-5]. Inhibitors of the three active sites of RAS have proven to be effective for the treatment of hypertension and congestive heart failure. Research efforts over the last decades have focused on the development of highly selective ANG II AT1 receptor blockers (ARBs) [6]. The DuPont group was the first to develop losartan (DuP 753), an orally effective angiotensin receptor blocker, which is metabolized in vivo to the more potent full antagonist EXP 3174 [7,8]. The discovery of losartan has stimulated the design of a large number of congeners [4,5]. Among them, eprosartan, irbesartan, candesartan, valsartan, olmesartan and azilsartan have been launched in the market [6,9-11]. Extensive Structure-Activity Relationships (SAR) and pharmacophore modeling studies [12] of ARBs, as well as the available data from literature, have illustrated the key elements required for the design of potent AT1 blockers [1]. Lipophilic substituents, such as the biphenylmethyl fragment substituted with an acidic moiety (tetrazole group, $\mathrm{CO}_{2} \mathrm{H}$ ) at the $N-1$ position of a heterocyclic ring and a linear alkyl group, providing an interaction with a hydrophobic pocket of the receptor, are required for potent antagonistic activity [6,13-17]. The DuPont group recommended a lipophilic and electron-withdrawing group such as a halogen atom, $\mathrm{CF}_{3}$, ethyl or pentafluoroethyl substituents at the $C-4$ of the imidazole ring and a small sized group such as $\mathrm{CH}_{2} \mathrm{OH}$ or $\mathrm{CO}_{2} \mathrm{H}$ at the $C-5$ capable of forming a hydrogen bond $[6,9,18]$.

Our recent work on the synthesis of AT1 receptor antagonists [18,19] indicated that 4(5)-butylimidazole-based analogues displayed significant antihypertensive activity. As a continuation of our studies [18-20], we report herein on the preparation of (E)-urocanic acid-based analogues, focusing our attention on the structural modifications on the imidazole ring which would possibly enhance potency. Consequently, we have designed using docking studies and synthesized a series of (E)-urocanic acid derivatives bearing the benzyl and the biphenylmethyl tetrazole moiety at the $N-1$ or $N-3$ position of the imidazole ring. Furthermore, these analogues bear the $(E)$-acrylic acid chain of (E)-urocanic acid as well as the corresponding saturated side chain at the $C$-4, mimicking the carboxyterminal region of the octapeptide ANG II [21,22] and a bulky lipophilic and electron-withdrawing group such as a halogen atom at the $C-5$ of the imidazole ring [6,9]. Additionally, some ARBs possessing two acidic groups, such as the tetrazole and the carboxyl group, have exhibited low oral bioavailability (BA) because of their highly polar character [6]. Thus, the rigid acrylic or the saturated acid side chain was masked by esterification resulting in the methyl ester or the bulky ester group (5-methyl-2-oxo-1,3-dioxol-4-yl)methyl or medoxomil [5,9], which is metabolized in vivo to the carboxyl moiety and may prove to be an effective structural element, emerging to compounds with improved BA. Finally, the reason for the shortening of the biphenyl group was to evaluate the ability of the tetrazole group to trigger activity located on a phenyl group instead of a biphenyl moiety and on the other hand to examine the ability of a single phenyl group to interact appropriately with the 
receptor [23]. Our synthetic approach included fast, efficient and regioselective reactions in high yields, allowing the facile introduction of the substituents on the imidazole nucleus. The synthesized analogues were finally tested for their AT1 receptor affinity using binding assays.

\section{Results and Discussion}

\subsection{Chemistry}

The intermediates $\mathbf{2}$ and $\mathbf{4}$ that were used to introduce the benzyl and the biphenylmethyl moiety to the imidazole ring were obtained according to reported methods as outlined in Scheme $1[8,18,24,25]$. In this case, protection of the tetrazole ring with the 2-chlorotrityl group by treatment with 2-chlorotrityl chloride $(\mathrm{ClTr}-\mathrm{Cl})$, followed by benzylic bromination provided the requisite alkylating agents 2 and 4 [18].

Scheme 1. Synthesis of the alkylating agents 2 and $\mathbf{4}[8,18,24,25]$.

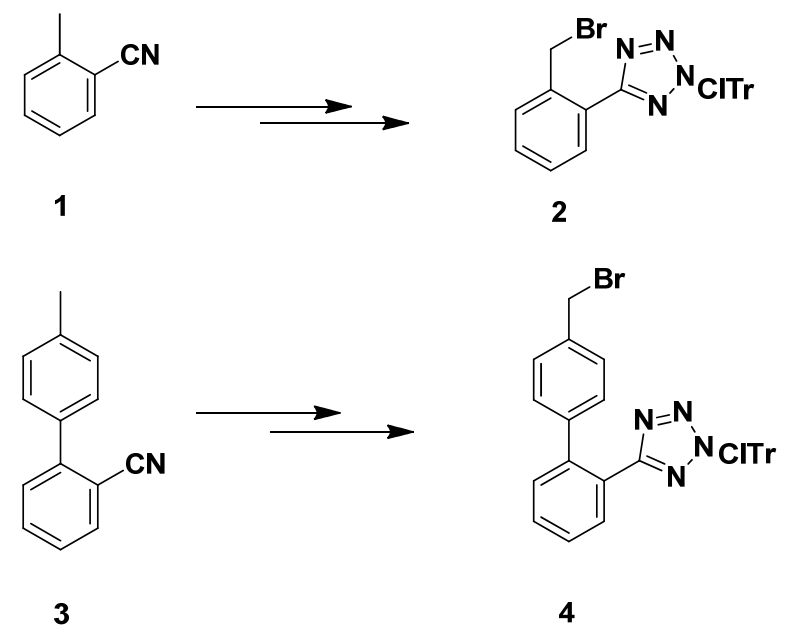

The preparation of $N$-benzyl imidazole derivatives $\mathbf{1 0 - 1 2}$ and 14 is depicted in Scheme 2 . $(E)$-Urocanic acid (5) was converted to the corresponding methyl ester $\mathbf{6}$ by esterification in dry methanol $(\mathrm{MeOH})[20,26]$. The ${ }^{1} \mathrm{H}-\mathrm{NMR}$ spectrum of $\mathbf{6}$ showed two singlet peaks at $\delta 7.78$ and 7.43 ppm corresponding to the H-2 and H-5 of the imidazole ring, respectively. Additionally, the two vinylic protons appeared as doublets at $\delta 6.44$ and $7.61(J=16.0 \mathrm{~Hz})$, respectively and the methoxy group at $3.78 \mathrm{ppm}$. Alkylation of the methyl ester 6 at the $N-1$ position of the imidazole ring with the benzyl alkylating agent $\mathbf{2}$, in the presence of sodium hydride $(\mathrm{NaH})$ in dry $N, N$-dimethylformamide (DMF), afforded 7 in 74\% yield. The ${ }^{1} \mathrm{H}-\mathrm{NMR}$ spectrum of 7 showed a singlet peak at $\delta 5.38$ assigned to the methylene protons. Catalytic hydrogenation $(\mathrm{Pd} / \mathrm{C})$ of the latter afforded the saturated derivative 8 in excellent yield (90\%). The ${ }^{1} \mathrm{H}-\mathrm{NMR}$ spectra of $\mathbf{8}$ showed two triplet peaks at $\delta 2.76$ and 2.56 $(J=7.2 \mathrm{~Hz})$ corresponding to the methylene protons of the saturated side chain. Alkaline hydrolysis [9] of the methyl esters 7 and $\mathbf{8}$ under mild conditions using a mixture of $\mathrm{KOH}$ in 1:1 $\mathrm{H}_{2} \mathrm{O}$ /dioxane, led to the corresponding acids $\mathbf{1 3}$ and $\mathbf{9}$, respectively. Removal of the ClTr group by treatment with $30 \%$ trifluoroacetic acid (TFA) in dichloromethane $\left(\mathrm{CH}_{2} \mathrm{Cl}_{2}\right)$, in the presence of triethylsilane $\left(\mathrm{Et} \mathrm{SiH}_{3}\right)$ as scavenger, provided the final analogues 10-12 and 14. 
Scheme 2. Synthesis of the target $N$-benzyl analogues $10,11,12,14^{\mathrm{a}}$.

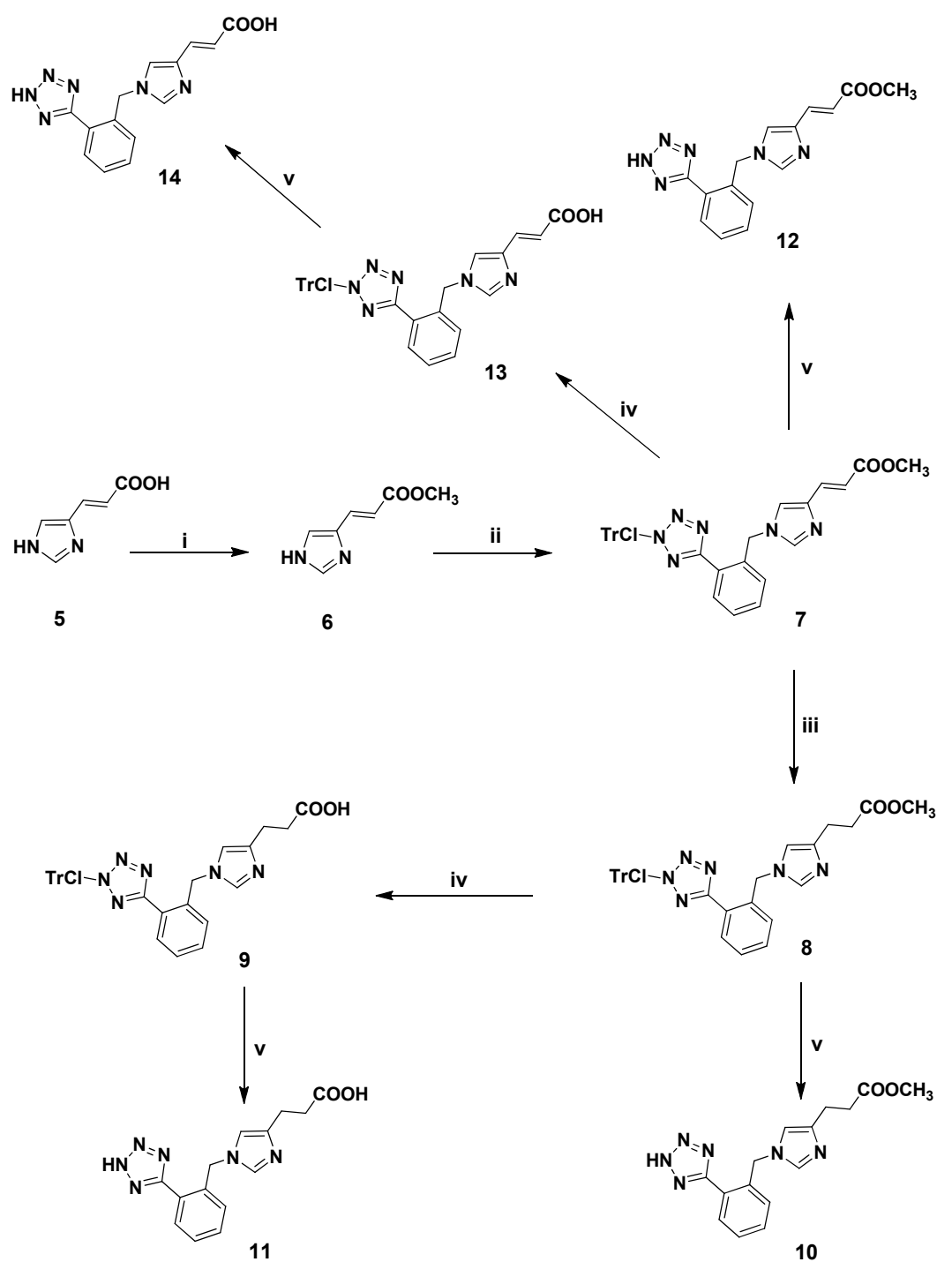

a Reagents and conditions: (i) $\mathrm{MeOH}$, anhydrous $\mathrm{Na}_{2} \mathrm{SO}_{4}$, conc. $\mathrm{H}_{2} \mathrm{SO}_{4}$, reflux, $24 \mathrm{~h}$; (ii) 2, $\mathrm{NaH}$ (powdered 95\%), dry DMF, $0{ }^{\circ} \mathrm{C}$ to $\mathrm{rt}, 4 \mathrm{~h}$; (iii) $\mathrm{H}_{2}, 10 \% \mathrm{Pd}-\mathrm{C}, \mathrm{MeOH}, \mathrm{rt}, 3 \mathrm{~h}$; (iv) $\mathrm{KOH}$, $\mathrm{H}_{2} \mathrm{O} /$ dioxane (1:1), rt, $3 \mathrm{~h}$; (v) $30 \%$ TFA in $\mathrm{CH}_{2} \mathrm{Cl}_{2}, \mathrm{Et}_{3} \mathrm{SiH}, \mathrm{rt}, 1 \mathrm{~h}$.

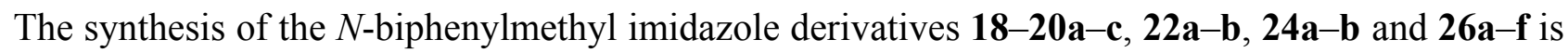
demonstrated in Scheme 3. Likewise, direct alkylation of the methyl ester $\mathbf{6}$ at the $\mathrm{N}-1$ position of the imidazole ring with the biphenylmethyl alkylating agent 4 afforded the 1,4-disubstituted analogue 15. The ${ }^{1} \mathrm{H}-\mathrm{NMR}$ data of $\mathbf{1 5}$ showed a singlet peak at $\delta 4.95$ due to the methylene protons of the alkylating moiety. Subsequently, hydrogenation of $\mathbf{1 5}$ in the presence of $10 \% \mathrm{Pd}-\mathrm{C}$ as catalyst in $\mathrm{MeOH}$, led to the intermediate 16. Halogenation of $\mathbf{1 6}$ at the $C-5$ position of the imidazole ring with the appropriate $N$-halosuccinimide (NXS, X $=\mathrm{Cl}, \mathrm{Br}, \mathrm{I}$ ) [18], afforded the halogenated derivatives 17a-c. The ${ }^{1} \mathrm{H}-\mathrm{NMR}$ spectra showed the absence of the H-5 signal of the imidazole ring at $6.47 \mathrm{ppm}$ appearing in 16. Saponification of the methyl esters $15-17 \mathbf{a}-\mathbf{c}$ was mediated by an aqueous solution of $\mathrm{KOH}$ in dioxane for $3 \mathrm{~h}$ at rt, to afford the corresponding acids 21a, 23a, 25a, 25c and 25e, respectively. The ${ }^{1} \mathrm{H}-\mathrm{NMR}$ spectra confirmed the absence of the methoxy group at 3.56-3.77 ppm. Treatment of the latter acids with medoxomil chloride (4-chloromethyl-5-methyl-2-oxo-1,3-dioxole) in the presence of 
potassium carbonate $\left(\mathrm{K}_{2} \mathrm{CO}_{3}\right)$ in dry $N, N$-dimethylacetamide (DMA), [9] furnished the esters $\mathbf{2 1 b}, \mathbf{2 3 b}$, 25b, 25d and 25f. The presence of the $-\mathrm{OCH}_{2}$ protons signal at $5.02-4.71 \mathrm{ppm}$ as well as the methyl protons signal at 2.02-2.16, unequivocally confirmed the introduction of the medoxomil group. Detritylation of the tetrazole group was accomplished by treatment with TFA in $\mathrm{CH}_{2} \mathrm{Cl}_{2}$, resulting in the target compounds 18-20a-c, 22a-b, 24a-b and 26a-f.

Scheme 3. Synthesis of the final $N$-biphenylmethyl analogues 18, 19, 20a-c, 22a-b, $24 a-b, 26 a-f^{a}$.
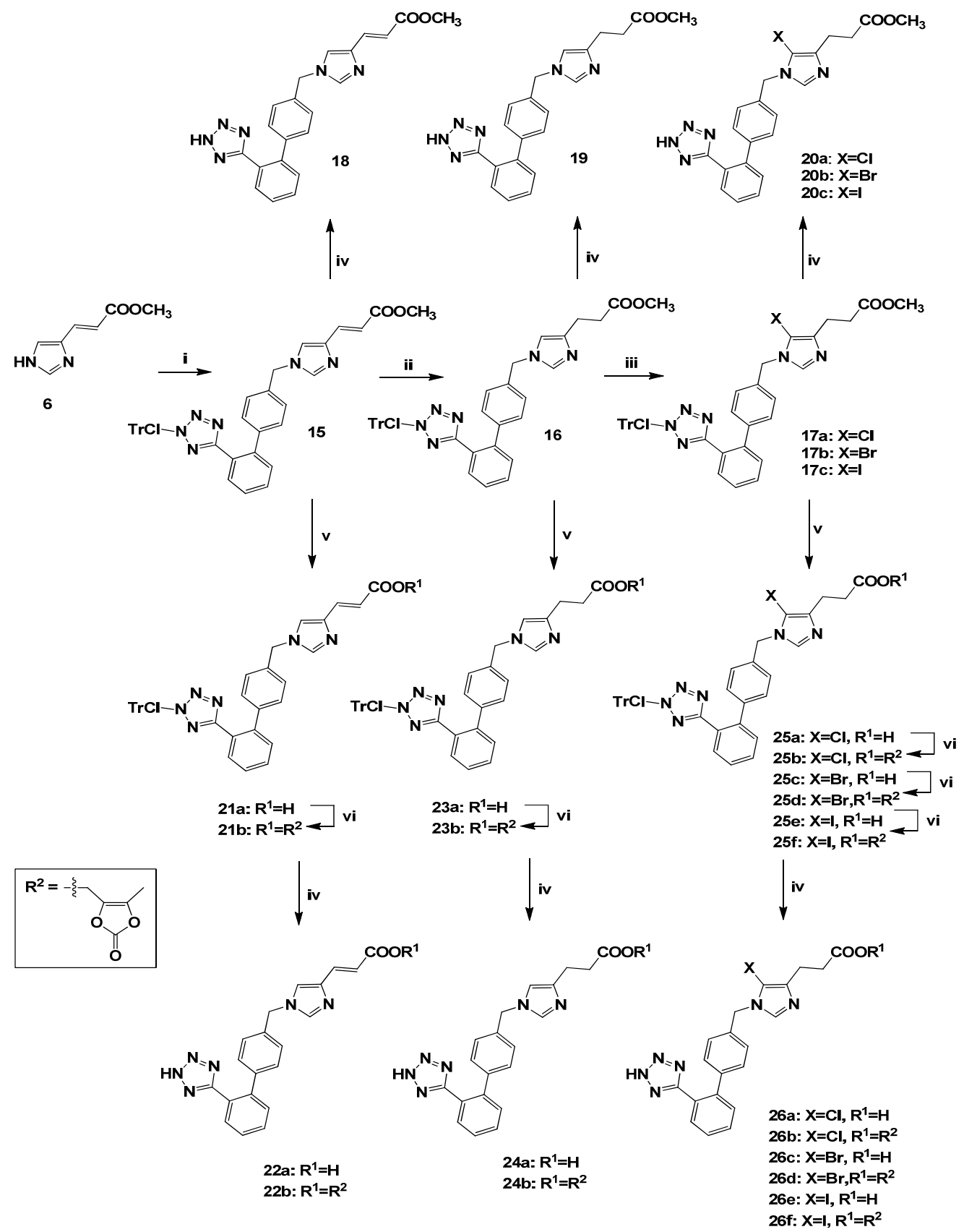

${ }^{a}$ Reagents and conditions: (i) 4, $\mathrm{NaH}$ (powdered 95\%), dry DMF, $0{ }^{\circ} \mathrm{C}$ to $\mathrm{rt}, 4 \mathrm{~h}$; (ii) $\mathrm{H}_{2}, 10 \%$ Pd-C, MeOH, rt, 3 h; (iii) NXS, X = Br, I in dry DMF or NCS in MeCN, $0{ }^{\circ} \mathrm{C}$ to rt, $4 \mathrm{~h}$; (iv) $30 \%$ TFA in $\mathrm{CH}_{2} \mathrm{Cl}_{2}, \mathrm{Et}_{3} \mathrm{SiH}, \mathrm{rt}, 1 \mathrm{~h}$; (v) $\mathrm{KOH}, \mathrm{H}_{2} \mathrm{O}$ /dioxane (1:1), rt, $3 \mathrm{~h}$; (vi) $\mathrm{K}_{2} \mathrm{CO}_{3}$, dry DMA, 4-chloromethyl-5-methyl-2-oxo-1,3-dioxole, rt, $4 \mathrm{~h}$. 
Finally, the preparation of the $N$-biphenylmethyl imidazole derivatives $\mathbf{3 0}$ and $\mathbf{3 2}$ is depicted in Scheme 4. Firstly, the imidazole ring was protected at the $N$-1 by the 2-(trimethylsilyl)ethoxymethyl (SEM) group using standard conditions [18,27,28]. Thus, treatment of the unsaturated methyl ester 6 with SEM-Cl in the presence of $\mathrm{NaH}$ in dry DMF, at ambient temperature for $2 \mathrm{~h}$, led to 27 in $78 \%$ yield. It is worth noting, that using the latter reaction conditions only the desired 1,4-regioisomer was formed, as indicated by HPLC and ${ }^{1} \mathrm{H}-\mathrm{NMR}$.

Scheme 4. Synthesis of the final $N$-biphenylmethyl substituted analogues $\mathbf{3 0}$ and $\mathbf{3 2}{ }^{\mathrm{a}}$.

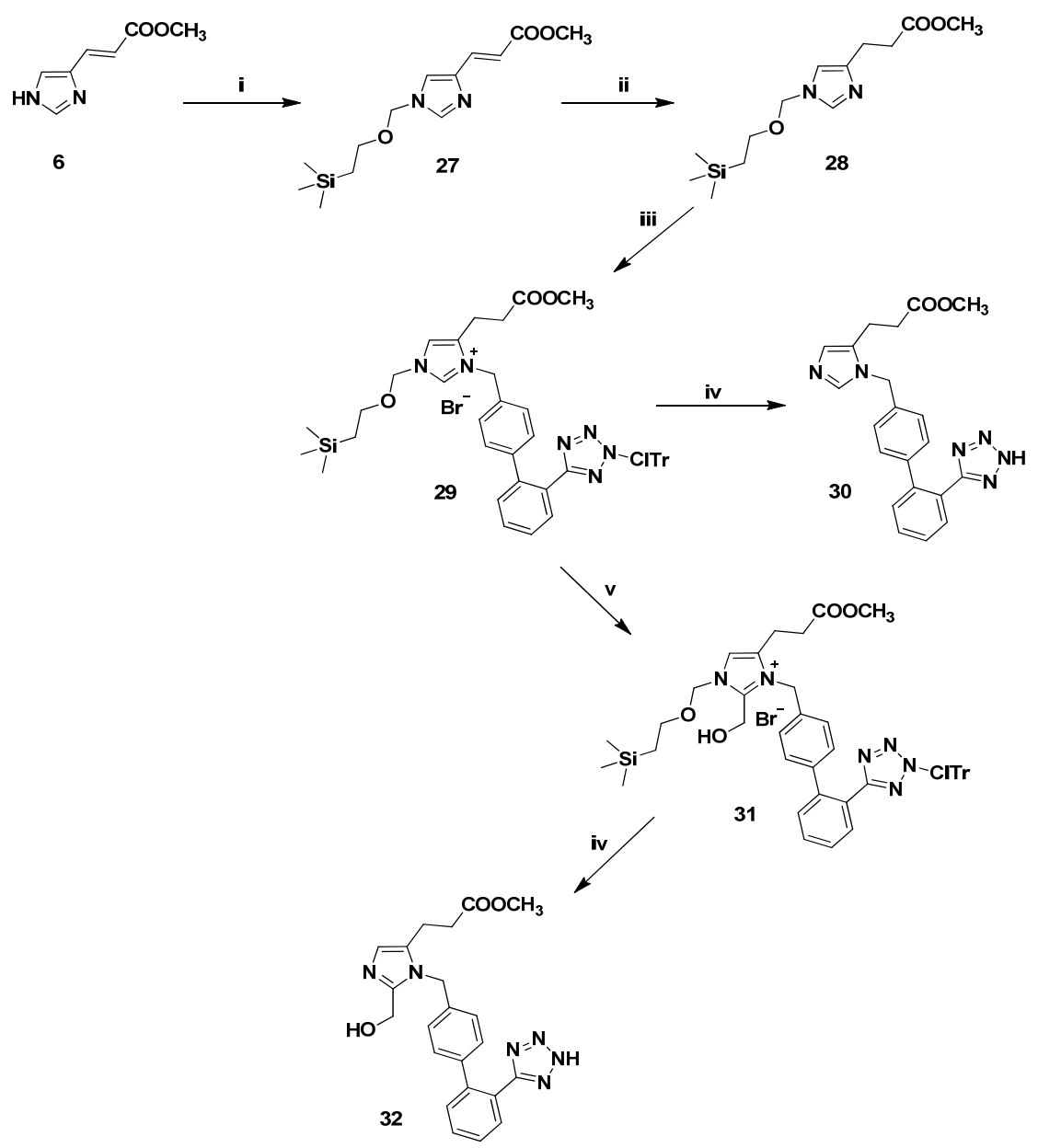

${ }^{a}$ Reagents and conditions: (i) SEM-Cl, NaH (powdered 95\%), dry DMF, $0{ }^{\circ} \mathrm{C}$ to rt, $2 \mathrm{~h}$; (ii) $\mathrm{H}_{2}$, $10 \% \mathrm{Pd}-\mathrm{C}, \mathrm{MeOH}, \mathrm{rt}, 3 \mathrm{~h}$; (iii) 4, $\mathrm{CH}_{2} \mathrm{Cl}_{2}$, reflux, $3 \mathrm{~h}$; (iv) $30 \% \mathrm{TFA}$ in $\mathrm{CH}_{2} \mathrm{Cl}_{2}, \mathrm{Et}_{3} \mathrm{SiH}, \mathrm{rt}, 1 \mathrm{~h}$; (v) $37 \%$ formalin, diisopropylethylamine, DMF, $85^{\circ} \mathrm{C}, 1 \mathrm{~h}$.

The resulting derivative 27 was subjected to hydrogenation in the presence of catalyst $10 \% \mathrm{Pd}-\mathrm{C}$ in $\mathrm{MeOH}$ to afford 28, in 91\% yield. Regioselective alkylation at the $N-3$ position was performed in the presence of the alkylating reagent 4 in $\mathrm{CH}_{2} \mathrm{Cl}_{2}$ under reflux for $3 \mathrm{~h}$, resulting in the intermediate salt 29 in high yield (81\%). Thus, the SEM group was proven to be an excellent choice for the protection of the $N-1$ followed by regioselective alkylation at the $N-3$ of the imidazole ring. At this point, we were ready to perform the introduction of the hydroxymethyl group at the $C-2$ of the imidazole ring of the alkylated analogue 29. According to our strategy [18], the hydroxymethylation was promptly carried out in a sealed tube by treatment with diisopropylethylamine and $37 \%$ formalin in DMF at $85{ }^{\circ} \mathrm{C}$ for $1 \mathrm{~h}$. The obtained residue was purified by column chromatography to afford the hydroxymethylated 
product 31 in excellent yield (91\%) and purity. The ${ }^{1} \mathrm{H}-\mathrm{NMR}$ spectrum of $\mathbf{3 1}$ showed the presence of a singlet peak at $4.72 \mathrm{ppm}$ due to the hydroxymethyl protons. Removal of the ClTr group by means of $30 \%$ TFA in $\mathrm{CH}_{2} \mathrm{Cl}_{2}$ and $\mathrm{Et}_{3} \mathrm{SiH}$ led to the 1,5-disubstituted imidazole analogues 30 and 32.

\subsection{Pharmacology}

The new synthesized analogues were evaluated in radioligand binding assay at a final concentration of $10^{-5} \mathrm{M}$. Although the used concentration was high enough, there were indications for moderate activity of the analogues $\mathbf{1 2}$ and $\mathbf{1 8}$. Competitive binding experiments revealed that the latter analogues caused $40.1 \%$ and $59.4 \%$ displacement of $\left[{ }^{125} \mathrm{I}\right]-\mathrm{Sar}^{1}-\mathrm{Ile}^{8}-\mathrm{ANG}$ II from the AT1 receptor, respectively, whereas losartan at the same conditions caused $100 \%$ displacement.

\subsection{Docking Studies}

The synthesized analogues have been rationalized based on their highest docking scores (Table 1). We notice that some of these analogues show higher scoring than losartan as reported in our previous paper [18].

Table 1. Highest Docking Scores of the synthesized analogues obtained with GLIDE/IFD ${ }^{\mathrm{a}}$.

\begin{tabular}{cc}
\hline Compounds & Docking Score \\
\hline Losartan & -12.114 \\
$\mathbf{1 0}$ & -9.625 \\
$\mathbf{1 2}$ & -10.276 \\
$\mathbf{1 8}$ & -12.089 \\
$\mathbf{1 9}$ & -11.395 \\
$\mathbf{2 0 a}$ & -10.397 \\
$\mathbf{2 0 b}$ & -11.099 \\
$\mathbf{2 0 c}$ & -11.899 \\
$\mathbf{2 2 a}$ & -11.577 \\
$\mathbf{2 2 b}$ & -14.401 \\
$\mathbf{2 4 a}$ & -12.321 \\
$\mathbf{2 4 b}$ & -13.570 \\
$\mathbf{2 6 a}$ & -10.577 \\
$\mathbf{2 6 b}$ & -13.463 \\
$\mathbf{2 6 c}$ & -9.404 \\
$\mathbf{2 6 d}$ & -13.047 \\
$\mathbf{2 6 e}$ & -13.168 \\
$\mathbf{2 6 f}$ & -12.017 \\
$\mathbf{3 0}$ & -12.281 \\
$\mathbf{3 2}$ & -11.012 \\
\hline${ }^{\mathrm{a}}$ Induced Fit Docking/XP module.
\end{tabular}

However, high scores could not rationalize the pharmacological results which showed that most of the synthesized analogues were inactive and only few of them showed moderate activity. In order to comprehend the pharmacological data, we have used as a template for comparison the putative 
bioactive conformation of losartan in the AT1 receptor presented by the pose of Figure 1a. Interestingly, the inactive compounds adopted losartan's orientation with poses of low scoring. The highest scoring orientations differed from that of losartan (Figure 1). As a result of this, the inactive compounds, even though docked in the same cavity, exerted different critical interactions that explain their inability to possess pharmacological activity. This is also applied with analogues $\mathbf{1 2}$ and $\mathbf{1 8}$ that showed $40.1 \%$ and $59.4 \%$ displacement of $\left[{ }^{125} \mathrm{I}\right]-\mathrm{Sar}^{1}-\mathrm{Ile}^{8}$-ANG II from the AT1 receptor (Figure 2).

Figure 1. Ligand interactions of $\mathbf{a}$ losartan; $\mathbf{b}$ and $\mathbf{c}$ analogue 12; $\mathbf{d}$ and $\mathbf{e}$ analogue $\mathbf{1 8}$ with the aminoacids of the active site of AT1; (b and $\mathbf{d}$ represent orientations of analogues $\mathbf{1 2}$ and 18 with the highest scoring and $\mathbf{c}$ and e. represent orientations with low scoring).

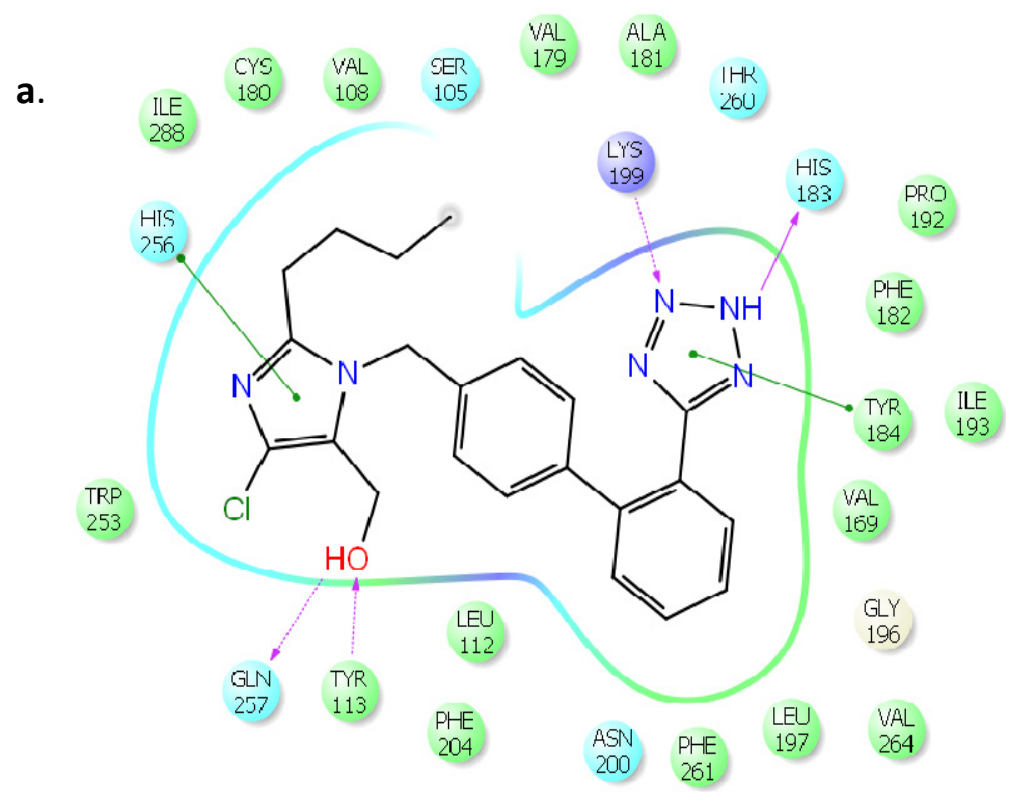

b.

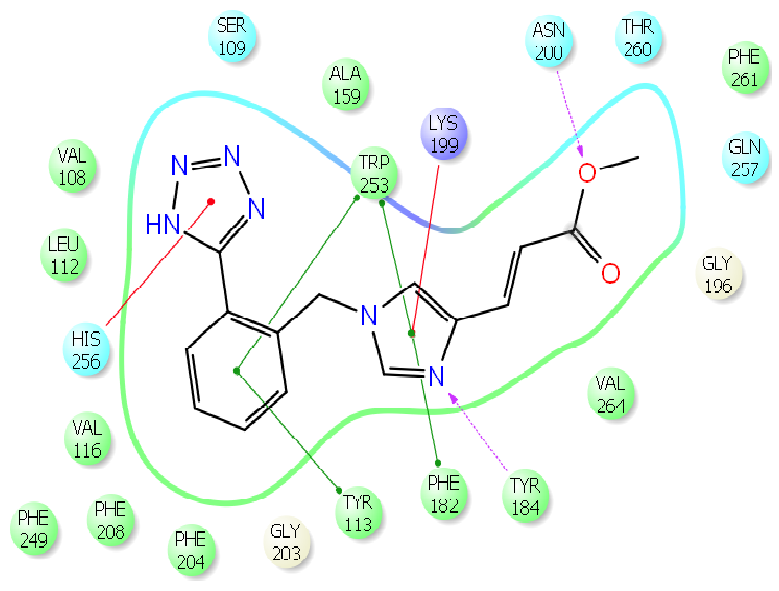

c. $\quad \begin{aligned} & \text { ALA } \\ & 291\end{aligned}$

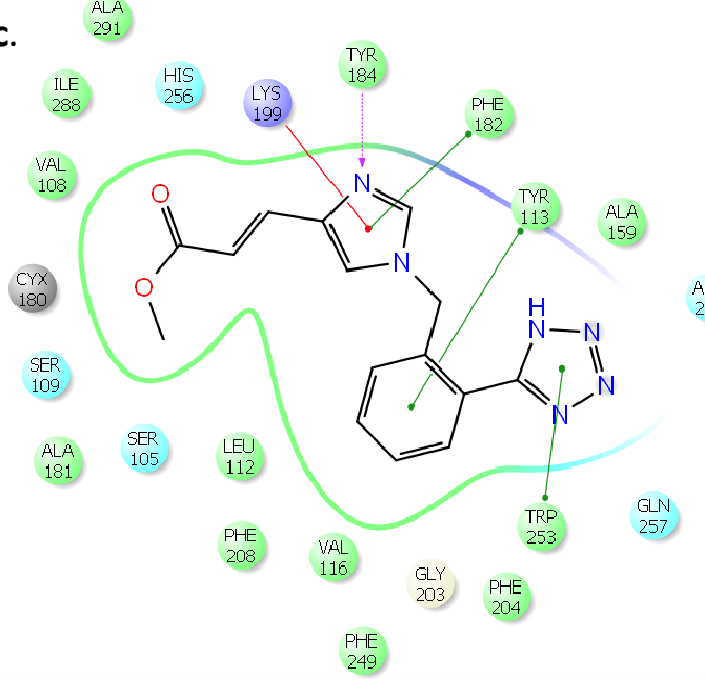


Figure 1. Cont.
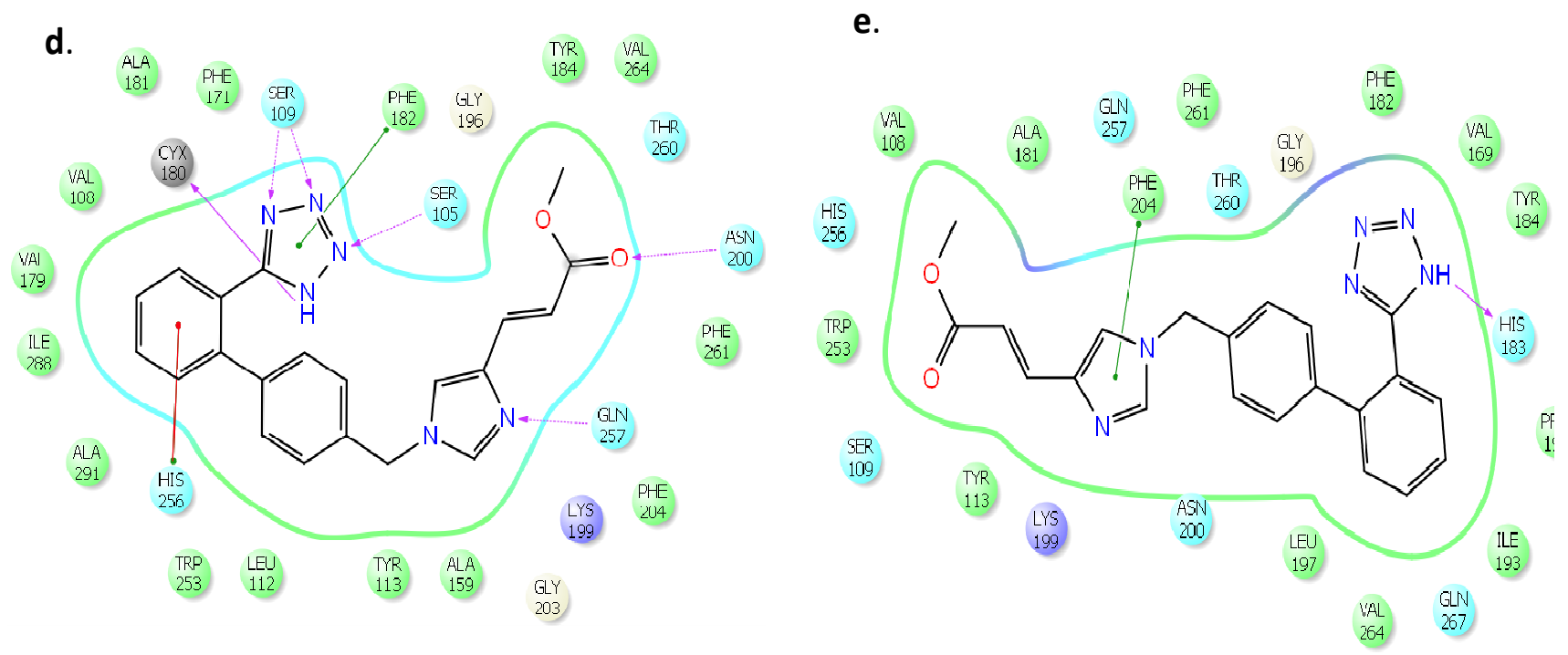

As it is shown in Figure 1b,d the poses that showed the highest scoring $(\mathbf{1 2},-10.276 \mathrm{kcal} / \mathrm{mol}$ and $18,-12.089 \mathrm{kcal} / \mathrm{mol}$ ), adopted orientations that did not match that of losartan. For example, analogue $\mathbf{1 8}$ is lacking the hydrogen bonding with Lys199 and both $\mathbf{1 2}$ and $\mathbf{1 8}$ are lacking the hydrogen bonding with His183, Gln257 and Tyr113. However, the poses that resembled the orientation of losartan (Figure 1c,e) showed low scorings $(\mathbf{1 2},-8.779 \mathrm{kcal} / \mathrm{mol}$ and $\mathbf{1 8},-5.217 \mathrm{kcal} / \mathrm{mol})$. This is attributed to the fact that both 12 and 18 cannot adopt the maximal critical interactions. For example, 18 forms only two hydrogen bondings (losartan forms four) and $\mathbf{1 2}$ only one. It appears that docking experiments could shed light on the required molecular interactions for drug activity only when pharmacological data were obtained.

Figure 2. a. Superimposition of losartan (green), with low scoring orientations of analogues 12 (orange) and $\mathbf{1 8}$ (yellow). b. with the highest scoring orientations.
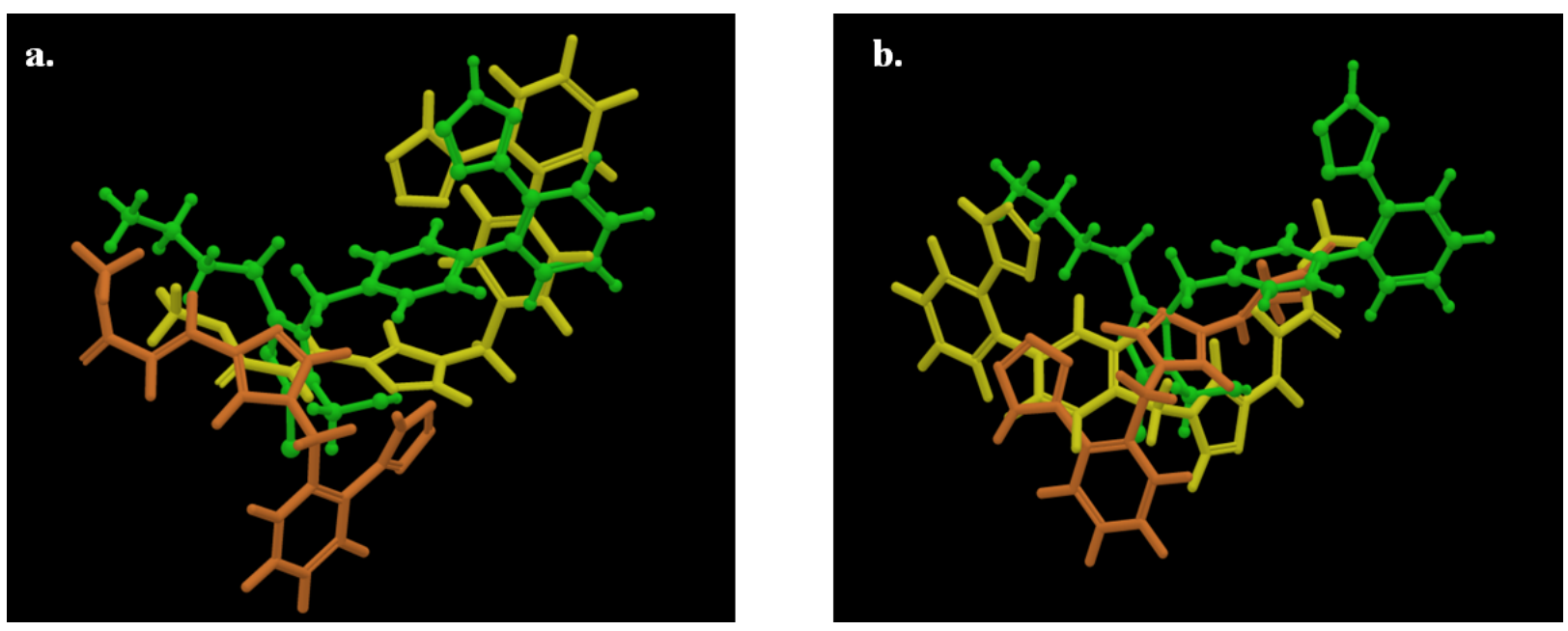


\section{Experimental}

\subsection{General}

Starting materials were purchased by Aldrich (Patras, Greece) and used as received. Hydrogenation reaction was carried out in a Parr hydrogenation apparatus equipped with a 4 L hydrogen tank. The ${ }^{1} \mathrm{H}-\mathrm{NMR}$ and ${ }^{13} \mathrm{C}-\mathrm{NMR}$ spectra were recorded on a Bruker Avance DPX spectrometer at $400.13 \mathrm{MHz}$ and $161.76 \mathrm{MHz}$, respectively. Chemical shifts are given in $\delta$ values (ppm) using tetramethylsilane as the internal standard and coupling constants $(J)$ are given in Hertz $(\mathrm{Hz})$. HPLC analysis was performed on an Alliance Waters 2695 equipped with a Waters 2996 Photodiode Array Detector UV-Vis, using the XBridge Waters $\mathrm{C} 18$ column $(4.6 \times 150 \mathrm{~mm}, 3.5 \mu \mathrm{m})$ as stationary phase and a gradient of $\mathrm{H}_{2} \mathrm{O} / \mathrm{MeCN}$ both containing $0.08 \%$ TFA as mobile phase. Electrospray-ionization mass spectra (ESI-MS) were obtained on a UPLC (ultra performance liquid chromatography) equipped with SQ detector Acquity ${ }^{\mathrm{TM}}$ by Waters. All reactions were carried out in anhydrous solvents. Analytical TLC was performed on silica gel $60 \mathrm{~F}_{254}$ plates (Merck, Germany) and visualized by UV irradiation and iodine. Silica gel $60 \mathrm{~N}$ (particle size $0.04-0.063 \mathrm{~mm}$ ) was used for column chomatography.

\subsection{Synthesis}

\subsubsection{General Procedure 1: Alkylation of the $(E)$-urocanic Methyl Esters at the N-1 Position}

To a solution of $6(2.0 \mathrm{~g}, 13.16 \mathrm{mmol})$ in dry DMF $(25 \mathrm{~mL})$, dry $\mathrm{NaH}$ (powdered 95\%, $0.35 \mathrm{~g}$, $14.47 \mathrm{mmol}$ ) was added and the resulting suspension was stirred for $30 \mathrm{~min}$ at $0{ }^{\circ} \mathrm{C}$ under nitrogen Then, $4(8.56 \mathrm{~g}, 14.48 \mathrm{mmol})$ was added in two portions and the mixture was stirred for $4 \mathrm{~h}$ at RT. The mixture was diluted in $\mathrm{H}_{2} \mathrm{O}$, extracted with $\mathrm{CH}_{2} \mathrm{Cl}_{2}$ and the organic phase was washed successively with $5 \% \mathrm{w} / \mathrm{v}$ citric acid, brine, dried over $\mathrm{Na}_{2} \mathrm{SO}_{4}$ and concentrated. The residue was purified by flash column chromatography (7:3 EtOAc:hexanes) to afford 15.

3.2.2. General Procedure 2: Catalytic Hydrogenation of the $N$-1 Alkylated (E)-Urocanic methyl Esters

A mixture of 15 (5.0 g, $7.54 \mathrm{mmol}), 10 \% \mathrm{w} / \mathrm{w} \mathrm{Pd}-\mathrm{C}(0.50 \mathrm{~g})$ in $\mathrm{MeOH}(20.0 \mathrm{~mL})$ was stirred under a hydrogen atmosphere ( 3 bar) at ambient temperature for $3 \mathrm{~h}$. The catalyst was filtered off by a Celite pad and the filtrate was concentrated in vacuo. The residue was purified by flash column chromatography (EtOAc) to provide $\mathbf{1 6 .}$

\subsubsection{General Procedure 3: Halogenation of the $N-1$ Alkylated Imidazole Derivatives}

To a solution of $16(1.0 \mathrm{~g}, 1.5 \mathrm{mmol})$ in dry DMF $(5.0 \mathrm{~mL})$ at $0{ }^{\circ} \mathrm{C}$ under nitrogen, NBS ( $0.29 \mathrm{~g}, 1.65 \mathrm{mmol})$ was added in three portions and the mixture was allowed to cool at room temperature. After $4 \mathrm{~h}$, the solvent was removed and the residue was purified by flash column chromatography (8:2, EtOAc:hexanes) to provide $\mathbf{1 7 b}$. 


\subsubsection{General Procedure 4: Alkaline Hydrolysis of the $N$-Substituted Imidazole Methyl Esters}

To a solution of $16(2.50 \mathrm{~g}, 3.75 \mathrm{mmol})$ in $\mathrm{H}_{2} \mathrm{O}$ /dioxane $(10.0 \mathrm{~mL}, 1: 1)$ was added fine powdered $\mathrm{KOH}(2.10 \mathrm{~g}, 37.50 \mathrm{mmol})$ and the resulting mixture was stirred at ambient temperature for $3 \mathrm{~h}$. The dioxane was removed by distillation in vacuo and to the residual solution was added $1 \mathrm{~N} \mathrm{HCl}$ to give a white precipitate 23a which was collected by vacuum filtration.

3.2.5. General Procedure 5: Esterification of the $N$-Substituted Imidazole Carboxylic Acids with the (5-Methyl-2-oxo-1,3-dioxol-4-yl)methyl or Medoxomil Group

To a solution of 23a $(2.0 \mathrm{~g}, 3.07 \mathrm{mmol})$ in dry DMA $(5.0 \mathrm{~mL})$ under nitrogen was added $\mathrm{K}_{2} \mathrm{CO}_{3}$ $(0.88 \mathrm{~g}, 6.41 \mathrm{mmol})$ and the mixture was stirred at ambient temperature for $30 \mathrm{~min}$. A solution of 4-chloromethyl-5-methyl-2-oxo-1,3-dioxole $(0.67 \mathrm{~g}, 4.56 \mathrm{mmol})$ in dry DMA was added dropwise and the resulting mixture was stirred for $4 \mathrm{~h}$. Then, the mixture was diluted with EtOAc and the organic phase was washed with $\mathrm{H}_{2} \mathrm{O}$, brine, dried over $\mathrm{Na}_{2} \mathrm{SO}_{4}$ and concentrated in vacuo. The crude product was purified by flash column chromatography (8:2 EtOAc:hexanes) to afford $\mathbf{2 3} \mathbf{b}$.

\subsubsection{General Procedure 6: Removal of the 2-Chlorotrityl Protecting Group}

To a solution of $16(0.50 \mathrm{~g}, 0.75 \mathrm{mmol})$ in $30 \%$ TFA in $\mathrm{CH}_{2} \mathrm{Cl}_{2}(2.0 \mathrm{~mL})$, TES $(0.10 \mathrm{~mL}, 0.63 \mathrm{mmol})$ was added dropwise and the resulting solution was stirred for $1 \mathrm{~h}$ at ambient temperature. The reaction mixture was concentrated and recrystallized from diethyl ether to afford $\mathbf{1 9}$.

\subsubsection{Synthesis of 1,4-Disubstituted Benzyl Analogues 10, 11, 12, 14}

N-(2-Chlorotrityl)-5-(2-bromobenzyl)tetrazole (2). Prepared from 1 according to the literature method $[8,18,24,25]$. Yield 78\%; $\mathrm{R}_{f}=0.46$ (2:8 EtOAc:hexanes); ESI-MS $(\mathrm{m} / \mathrm{z}): 238.27\left(\mathrm{M}+\mathrm{H}^{+}-\mathrm{ClTr}\right), 277.78$ $(\mathrm{ClTr}) ;{ }^{1} \mathrm{H}-\mathrm{NMR}\left(\mathrm{CDCl}_{3}\right): \delta 8.22-8.18(\mathrm{~m}, 1 \mathrm{H}), 7.51-7.32(\mathrm{~m}, 12 \mathrm{H}), 7.25-7.21(\mathrm{~m}, 4 \mathrm{H}), 6.86(\mathrm{~d}, 1 \mathrm{H}$, $J=7.6 \mathrm{~Hz}), 4.92(\mathrm{~s}, 2 \mathrm{H}) ;{ }^{13} \mathrm{C}-\mathrm{NMR}\left(\mathrm{CDCl}_{3}\right): \delta 162.90,141.28,136.87,131.64,130.46,129.96$, 128.94, 128.42, 127.96, 127.84, 127.29, 126.43, 83.45, 32.42. Anal. Calcd for $\mathrm{C}_{27} \mathrm{H}_{20} \mathrm{~N}_{4} \mathrm{ClBr}(\%)$ : C: 62.87; H: 3.91; N: 10.86. Found (\%): C: 62.99; H: 4.00; N: 10.54.

Methyl 3-(1H-imidazol-4-yl)acrylate (6). Prepared from 5 according to the literature method [20,26]. Yield 95\%; M.p. 92-94 ${ }^{\circ} \mathrm{C}$; $\mathrm{R}_{f}=0.42\left(9: 1 \mathrm{CHCl}_{3}: \mathrm{MeOH}\right)$; ESI-MS $(\mathrm{m} / \mathrm{z}): 153.22\left(\mathrm{M}+\mathrm{H}^{+}\right)$; ${ }^{1} \mathrm{H}-\mathrm{NMR}$ $\left(\mathrm{CD}_{3} \mathrm{OD}\right): \delta 7.78(\mathrm{~s}, 1 \mathrm{H}), 7.61(\mathrm{~d}, 1 \mathrm{H}, J=16.0 \mathrm{~Hz}), 7.43(\mathrm{~s}, 1 \mathrm{H}), 6.44(\mathrm{~d}, 1 \mathrm{H}, J=16.0 \mathrm{~Hz}), 3.78$ (s, $3 \mathrm{H}) ;{ }^{13} \mathrm{C}-\mathrm{NMR}\left(\mathrm{CDCl}_{3}\right): \delta 168.14,137.43,135.25,134.35,124.0,114.35,50.61$. Anal. Calcd for $\mathrm{C}_{7} \mathrm{H}_{8} \mathrm{~N}_{2} \mathrm{O}_{2}(\%)$ : C: 55.26; H: 5.30; N: 18.41. Found (\%): C: 55.21; H: 5.39; N: 18.37. All data were consistent with literature [26].

(E)-Methyl 1-[[1-[[N-(2-chlorotrityl)]-1H-tetrazol-5-yl]phenyl-2-yl]methyl]imidazole-4-acrylate (7). General procedure 1 was employed for the preparation of 7 using 2 as alkylating agent. Yield 74\%; $\mathrm{R}_{f}=0.38$ (8:2 EtOAc:hexanes); ESI-MS $(\mathrm{m} / \mathrm{z}): 587.27\left(\mathrm{M}+\mathrm{H}^{+}\right), 309.37\left(\mathrm{M}+\mathrm{H}^{+}-\mathrm{ClTr}\right), 277.88(\mathrm{ClTr})$; ${ }^{1} \mathrm{H}-\mathrm{NMR}\left(\mathrm{CDCl}_{3}\right): \delta 8.28(\mathrm{dd}, 1 \mathrm{H}, J=2.0,7.6 \mathrm{~Hz}), 7.53-7.10(\mathrm{~m}, 19 \mathrm{H}), 6.76(\mathrm{~s}, 1 \mathrm{H}), 6.48(\mathrm{~d}, 1 \mathrm{H}$, 
$J=15.6 \mathrm{~Hz}), 5.38(\mathrm{~s}, 2 \mathrm{H}), 3.77(\mathrm{~s}, 3 \mathrm{H})$. Anal. Calcd for $\mathrm{C}_{34} \mathrm{H}_{27} \mathrm{~N}_{6} \mathrm{O}_{2} \mathrm{Cl}(\%): \mathrm{C}: 74.02 ; \mathrm{H}: 4.30$; N: 13.28. Found (\%): C: 73.96; H: 4.22; N: 13.32.

Methyl 1-[[1-[[N-(2-chlorotrityl)]-1H-tetrazol-5-yl]phenyl-2-yl]methyl]imidazole-4-propanoate (8). General procedure 2 was employed for the preparation of 8. Yield 90\%; $\mathrm{R}_{f}=0.45\left(9: 1 \mathrm{CHCl}_{3}: \mathrm{MeOH}\right)$; ESI-MS $(\mathrm{m} / \mathrm{z}): 589.17\left(\mathrm{M}+\mathrm{H}^{+}\right), 311.45\left(\mathrm{M}+\mathrm{H}^{+}-\mathrm{ClTr}\right), 277.38(\mathrm{ClTr}) ;{ }^{1} \mathrm{H}-\mathrm{NMR}\left(\mathrm{CDCl}_{3}\right): \delta 8.18-8.15$ (m, 1H), 7.42-7.26 (m, 9H), 7.07-6.99 (m, 9H), $6.37(\mathrm{~s}, 1 \mathrm{H}), 5.27(\mathrm{~s}, 2 \mathrm{H}), 3.57(\mathrm{~s}, 3 \mathrm{H}), 2.76(\mathrm{t}, 2 \mathrm{H}$, $J=7.2 \mathrm{~Hz}), 2.56(\mathrm{t}, 2 \mathrm{H}, J=7.2 \mathrm{~Hz})$. Anal. Calcd for $\mathrm{C}_{34} \mathrm{H}_{29} \mathrm{~N}_{6} \mathrm{O}_{2} \mathrm{Cl}(\%)$ : C: 69.32; $\mathrm{H}: 4.96$; N: 14.27. Found (\%): C: $69.45 ; \mathrm{H}: 4.88 ; \mathrm{N}: 14.32$.

1-[[1-[[N-(2-Chlorotrityl)]-1H-tetrazol-5-yl]phenyl-2-yl]methyl]imidazole-4-propanoic acid (9). General procedure 4 was employed for the preparation of 9. Yield 93\%; $\mathrm{R}_{f}=0.31(8.5: 1.5$ $\left.\mathrm{CHCl}_{3}: \mathrm{MeOH}\right)$; ESI-MS $(\mathrm{m} / \mathrm{z}): 576.19\left(\mathrm{M}+\mathrm{H}^{+}\right), 299.63\left(\mathrm{M}+\mathrm{H}^{+}-\mathrm{ClTr}\right), 277.77$ (ClTr); ${ }^{1} \mathrm{H}-\mathrm{NMR}$ $\left(\mathrm{CDCl}_{3}\right): \delta 8.31(\mathrm{~d}, 1 \mathrm{H}, J=7.2 \mathrm{~Hz}), 7.54-7.11(\mathrm{~m}, 17 \mathrm{H}), 6.85(\mathrm{~d}, 1 \mathrm{H}, J=7.6 \mathrm{~Hz}), 6.42(\mathrm{~s}, 1 \mathrm{H}), 5.40$ (s, 2H), 2.73 (bs, 2H), 2.65 (bs, 2H). Anal. Calcd for $\mathrm{C}_{33} \mathrm{H}_{27} \mathrm{~N}_{6} \mathrm{O}_{2}(\%)$ : C: 68.92; H: 4.73; N: 14.61. Found (\%): C: 68.92; H: 4.73; N: 14.61.

Methyl 1-[[1-(1H-tetrazol-5-yl)phenyl-2-yl]methyl]imidazole-4-propanoate (10). General procedure 6 was employed for the preparation of 10. Yield 91\%; $\mathrm{R}_{f}=0.48\left(8: 2 \mathrm{CHCl}_{3}: \mathrm{MeOH}\right)$; ESI-MS $(\mathrm{m} / \mathrm{z})$ : $313.34\left(\mathrm{M}+\mathrm{H}^{+}\right) ;{ }^{1} \mathrm{H}-\mathrm{NMR}\left(\mathrm{CD}_{3} \mathrm{OD}\right): \delta 8.80(\mathrm{~s}, 1 \mathrm{H}), 7.96-7.93(\mathrm{~m}, 1 \mathrm{H}), 7.64-7.60(\mathrm{~m}, 3 \mathrm{H}), 7.33(\mathrm{~s}$, $1 \mathrm{H}), 5.75(\mathrm{~s}, 2 \mathrm{H}), 3.65(\mathrm{~s}, 3 \mathrm{H}), 2.94(\mathrm{t}, 2 \mathrm{H}, J=7.2 \mathrm{~Hz}), 2.69(\mathrm{t}, 2 \mathrm{H}, J=7.2 \mathrm{~Hz})$. Anal. Calcd for $\mathrm{C}_{15} \mathrm{H}_{16} \mathrm{~N}_{6} \mathrm{O}_{2} \cdot \mathrm{CF}_{3} \mathrm{COOH}(\%)$ : C: 47.89; H: 4.02; N: 19.71. Found (\%): C: 47.78; H: 3.95; N: 19.87.

1-[[1-(1H-Tetrazol-5-yl)phenyl-2-yl]methyl]imidazole-4-propanoic acid (11). General procedure 6 was employed for the preparation of 11. Yield 88\%; $\mathrm{R}_{f}=0.37\left(4: 1: 1 n\right.$-butanol:acetic acid: $\left.\mathrm{H}_{2} \mathrm{O}\right)$; ESI-MS (m/z): $297.24\left(\mathrm{M}+\mathrm{H}^{+}\right)$; ${ }^{1} \mathrm{H}-\mathrm{NMR}\left(\mathrm{CD}_{3} \mathrm{OD}\right): \delta 8.83(\mathrm{~s}, 1 \mathrm{H}), 7.97-7.93(\mathrm{~m}, 1 \mathrm{H}), 7.65-7.59(\mathrm{~m}$, $3 \mathrm{H}), 7.36(\mathrm{~s}, 1 \mathrm{H}), 5.76(\mathrm{~s}, 2 \mathrm{H}), 2.93(\mathrm{t}, 2 \mathrm{H}, J=7.2 \mathrm{~Hz}), 2.66(\mathrm{t}, 2 \mathrm{H}, J=7.2 \mathrm{~Hz})$. Anal. Calcd for $\mathrm{C}_{14} \mathrm{H}_{14} \mathrm{~N}_{6} \mathrm{O}_{2} \cdot \mathrm{CF}_{3} \mathrm{COOH}(\%)$ : C: 46.11; H: 3.67; N: 20.38. Found (\%): C: 46.02; H: 3.57; N: 20.48.

(E)-Methyl 1-[[1-(1H-tetrazol-5-yl)phenyl-2-yl]methyl]imidazole-4-acrylate (12). General procedure 6 was employed for the preparation of 12 . Yield 95\%; $\mathrm{R}_{f}=0.48\left(8: 2 \mathrm{CHCl}_{3}: \mathrm{MeOH}\right)$; ESI-MS $(\mathrm{m} / z)$ : $311.24\left(\mathrm{M}+\mathrm{H}^{+}\right) ;{ }^{1} \mathrm{H}-\mathrm{NMR}\left(\mathrm{DMSO}-d_{6}\right): \delta 8.46(\mathrm{~s}, 1 \mathrm{H}), 7.90(\mathrm{~s}, 1 \mathrm{H}), 7.79(\mathrm{~s}, 1 \mathrm{H}), 7.62(\mathrm{bs}, 2 \mathrm{H}), 7.58$ (m, 4H), $7.50(\mathrm{~d}, 1 \mathrm{H}, J=15.6 \mathrm{~Hz}), 7.34(\mathrm{~s}, 1 \mathrm{H}), 6.46(\mathrm{~d}, 1 \mathrm{H}, J=15.6 \mathrm{~Hz}), 5.68(\mathrm{~s}, 2 \mathrm{H}), 3.78(\mathrm{~s}, 3 \mathrm{H})$. Anal. Calcd for $\mathrm{C}_{15} \mathrm{H}_{14} \mathrm{~N}_{6} \mathrm{O}_{2} \cdot \mathrm{CF}_{3} \mathrm{COOH}(\%)$ : C: 48.12; H: 3.56; N: 19.81. Found (\%): C: 48.22; H: $3.47 ; \mathrm{N}: 19.71$.

(E)-1-[[1-[[N-(2-Chlorotrityl)]-1H-tetrazol-5-yl]phenyl-2-yl]methyl]imidazole-4-acrylic acid (13). General procedure 4 was employed for the preparation of 13. Yield 95\%; $\mathrm{R}_{f}=0.30(9: 1$ $\left.\mathrm{CHCl}_{3}: \mathrm{MeOH}\right)$; ESI-MS $(\mathrm{m} / \mathrm{z}): 574.15\left(\mathrm{M}+\mathrm{H}^{+}\right), 297.54\left(\mathrm{M}+\mathrm{H}^{+}-\mathrm{ClTr}\right), 277.78$ (ClTr); ${ }^{1} \mathrm{H}-\mathrm{NMR}$ $\left(\mathrm{CDCl}_{3}\right): \delta 8.28(\mathrm{~d}, 2 \mathrm{H}, J=5.6 \mathrm{~Hz}), 7.52-7.23(\mathrm{~m}, 12 \mathrm{H}), 7.10(\mathrm{~d}, 6 \mathrm{H}, J=5.6 \mathrm{~Hz}), 6.74(\mathrm{bs}, 1 \mathrm{H}), 6.44$ $(\mathrm{d}, 1 \mathrm{H}, J=15.6 \mathrm{~Hz}), 5.36(\mathrm{~s}, 2 \mathrm{H})$. Anal. Calcd for $\mathrm{C}_{33} \mathrm{H}_{25} \mathrm{~N}_{6} \mathrm{O}_{2} \mathrm{Cl}(\%)$ : C: 69.17; H: 4.40; N: 14.67 . Found (\%): C: 69.11; H: 4.29; N: 14.72. 
(E)-1-[[1-(1H-Tetrazol-5-yl)phenyl-2-yl]methyl]imidazole-4-acrylic acid (14). General procedure 6 was employed for the preparation of 14. Yield 95\%; $\mathrm{R}_{f}=0.50\left(4: 1: 1 n\right.$-butanol:acetic acid: $\left.\mathrm{H}_{2} \mathrm{O}\right)$; ESI-MS (m/z): $297.30\left(\mathrm{M}+\mathrm{H}^{+}\right) ;{ }^{1} \mathrm{H}-\mathrm{NMR}\left(\mathrm{CD}_{3} \mathrm{OD}\right): \delta 8.85(\mathrm{~s}, 1 \mathrm{H}), 7.98-7.95(\mathrm{~m}, 1 \mathrm{H}), 7.84(\mathrm{~s}, 1 \mathrm{H})$, 7.72-7.64 (m, 3H), $7.48(\mathrm{~d}, 1 \mathrm{H}, J=16.0 \mathrm{~Hz}), 6.49(\mathrm{~d}, 1 \mathrm{H}, J=16.0 \mathrm{~Hz}), 5.80(\mathrm{~s}, 2 \mathrm{H})$. Anal. Calcd for $\mathrm{C}_{14} \mathrm{H}_{12} \mathrm{~N}_{6} \mathrm{O}_{2} \cdot \mathrm{CF}_{3} \mathrm{COOH}(\%)$ : C: 48.12; H: 3.56; N: 19.81. Found (\%): C: 48.22; H: 3.47; N: 19.71.

\subsubsection{Synthesis of 1,4-Disubstituted biphenylmethyl analogues 18, 19, 20a-c, 22a-b, 24a-b, 26a-f}

N-(2-Chlorotrityl)-5-[4'-(bromomethyl)biphenyl-2-yl]tetrazole (4). Prepared from 3 according to the literature method [8,19,25,26]. Yield 80\%; M.p. 155-157 ${ }^{\circ} \mathrm{C} ; \mathrm{R}_{f}=0.33$ (1.5:8.5 EtOAc:hexanes); ESI-MS (m/z): $315.48\left(\mathrm{M}+\mathrm{H}^{+}\right.$-ClTr), $277.22(\mathrm{ClTr}) ;{ }^{1} \mathrm{H}-\mathrm{NMR}\left(\mathrm{CDCl}_{3}\right): \delta$ 8.02-7.89 (m, 1H), $7.60-6.87(\mathrm{~m}, 20 \mathrm{H}), 6.79(\mathrm{dd}, 1 \mathrm{H}, J=1.5,8.0 \mathrm{~Hz}), 4.43(\mathrm{~s}, 2 \mathrm{H}) ;{ }^{13} \mathrm{C}-\mathrm{NMR}\left(\mathrm{CDCl}_{3}\right): \delta 163.95,141.63$, $141.47,140.64,139.49,136.34,132.13,131.75,130.79,130.53,130.15,129.69,128.87,128.66$, 127.97, 127.91, 126.68, 81.96, 33.45. Anal. Calcd for $\mathrm{C}_{33} \mathrm{H}_{24} \mathrm{~N}_{4} \mathrm{ClBr}(\%)$ : C: 66.96; H: 4.09; N: 9.47. Found (\%): C: 66.88; H: 4.15; N: 9.42.

(E)-Methyl 1-[[2'-[[N-(2-chlorotrityl)]-1H-tetrazol-5-yl] biphenyl-4-yl]methyl]imidazole-4-acrylate (15). General procedure 1 was employed for the preparation of $\mathbf{1 5}$ using 4 as alkylating agent. Yield 73\%; $\mathrm{R}_{f}=0.50$ (EtOAc); ESI-MS (m/z): $664.19\left(\mathrm{M}+\mathrm{H}^{+}\right), 387.29\left(\mathrm{M}+\mathrm{H}^{+}-\mathrm{ClTr}\right), 277.38$ (ClTr); ${ }^{1} \mathrm{H}-\mathrm{NMR}\left(\mathrm{CDCl}_{3}\right): \delta 8.28(\mathrm{dd}, 1 \mathrm{H}, J=1.8,7.6 \mathrm{~Hz}), 7.53-7.12(\mathrm{~m}, 22 \mathrm{H}), 6.84(\mathrm{~m}, 2 \mathrm{H}), 6.54(\mathrm{~d}, 1 \mathrm{H}$, $J=15.6 \mathrm{~Hz}), 4.95$ (s, 2H), 3.77 (s, 3H); ${ }^{13} \mathrm{C}-\mathrm{NMR}\left(\mathrm{CDCl}_{3}\right): \delta 168.20,137.43,162.87,140.48,139.14$, $138.89,138.22,136.33,134.05,132.22,131.84,130.99,130.54,129.29,128.86,128.30,126.97$, 126.31, 122.18, 115.53, 82.66, 51.63, 49.53. Anal. Calcd for $\mathrm{C}_{40} \mathrm{H}_{31} \mathrm{~N}_{6} \mathrm{O}_{2} \mathrm{Cl}(\%)$ : C: 72.44; $\mathrm{H}: 4.71$; N: 12.67. Found (\%): C: $72.38 ; \mathrm{H}: 4.77 ; \mathrm{N}: 12.62$.

Methyl 1-[[2'-[[N-(2-chlorotrityl)]-1H-tetrazol-5-yl]biphenyl-4-yl]methyl]imidazole-4-propanoate (16). General procedure 2 was employed for the preparation of 16 . Yield $88 \% ; \mathrm{R}_{f}=0.55(9: 1$ $\left.\mathrm{CHCl}_{3}: \mathrm{MeOH}\right)$; ESI-MS (m/z): $666.19\left(\mathrm{M}+\mathrm{H}^{+}\right), 388.39\left(\mathrm{M}+\mathrm{H}^{+}-\mathrm{ClTr}\right), 277.77$ (ClTr); ${ }^{1} \mathrm{H}-\mathrm{NMR}$ $\left(\mathrm{CDCl}_{3}\right): \delta 7.98(\mathrm{dd}, 1 \mathrm{H}, J=2.0,7.6 \mathrm{~Hz}), 7.53-7.12(\mathrm{~m}, 14 \mathrm{H}), 6.89-6.83(\mathrm{~m}, 7 \mathrm{H}), 6.72(\mathrm{dd}, 1 \mathrm{H}$, $J=1.2,8.0 \mathrm{~Hz}), 6.47(\mathrm{~s}, 1 \mathrm{H}), 4.87(\mathrm{~s}, 2 \mathrm{H}), 3.65(\mathrm{~s}, 3 \mathrm{H}), 2.84(\mathrm{t}, 2 \mathrm{H}, J=7.6 \mathrm{~Hz}), 2.64(\mathrm{t}, 2 \mathrm{H}$, $J=7.6 \mathrm{~Hz}$ ). Anal. Calcd for $\mathrm{C}_{40} \mathrm{H}_{33} \mathrm{~N}_{6} \mathrm{O}_{2} \mathrm{Cl}(\%)$ : C: 72.23; $\mathrm{H}: 5.00 ; \mathrm{N}: 12.63$. Found (\%): C: 72.18; $\mathrm{H}$ : $5.09 ; \mathrm{N}: 12.57$.

Methyl 5-chloro-1-[[2'-[[N-(2-chlorotrityl)]-1H-tetrazol-5-yl]biphenyl-4-yl]methyl]imidazole-4propanoate (17a). General procedure 3 was employed for the preparation of 17a using NCS in MeCN. Yield 85\%; $\mathrm{R}_{f}=0.49$ (EtOAc); ESI-MS $(\mathrm{m} / \mathrm{z}): 700.59\left(\mathrm{M}+\mathrm{H}^{+}\right), 422.86\left(\mathrm{M}+\mathrm{H}^{+}-\mathrm{ClTr}\right), 277.77(\mathrm{ClTr})$; ${ }^{1} \mathrm{H}-\mathrm{NMR}\left(\mathrm{CDCl}_{3}\right): \delta 7.97(\mathrm{dd}, 1 \mathrm{H}, J=1.2,7.2 \mathrm{~Hz}), 7.52-7.14(\mathrm{~m}, 15 \mathrm{H}), 6.90-6.84(\mathrm{~m}, 6 \mathrm{H}), 6.72(\mathrm{~d}$, $1 \mathrm{H}, J=8.0 \mathrm{~Hz}), 4.89(\mathrm{~s}, 2 \mathrm{H}), 3.68(\mathrm{~s}, 3 \mathrm{H}), 2.87(\mathrm{t}, 2 \mathrm{H}, J=7.6 \mathrm{~Hz}), 2.70(\mathrm{t}, 2 \mathrm{H}, J=7.6 \mathrm{~Hz})$. $\mathrm{C}_{40} \mathrm{H}_{32} \mathrm{Cl}_{2} \mathrm{~N}_{6} \mathrm{O}_{2}$. Anal. Calcd for $\mathrm{C}_{40} \mathrm{H}_{32} \mathrm{~N}_{6} \mathrm{O}_{2} \mathrm{Cl}_{2}$ (\%): C: 68.67; H: 4.61; N: 12.01. Found (\%): C: 67.92; H: 4.78; N: 11.84 .

Methyl 5-bromo-1-[[2'-[[N-(2-chlorotrityl)]-1H-tetrazol-5-yl]biphenyl-4-yl]methyl]imidazole-4propanoate $(\mathbf{1 7 b})$. General procedure 3 was employed for the preparation of $\mathbf{1 7 b}$ using NBS in DMF. 
Yield 68\%; $\mathrm{R}_{f}=0.47$ (EtOAc); ESI-MS $(\mathrm{m} / \mathrm{z}): 745.54\left(\mathrm{M}+\mathrm{H}^{+}\right), 467.80\left(\mathrm{M}+\mathrm{H}^{+}-\mathrm{ClTr}\right), 277.80(\mathrm{ClTr})$; ${ }^{1} \mathrm{H}-\mathrm{NMR}\left(\mathrm{CDCl}_{3}\right): \delta 7.84(\mathrm{dd}, 1 \mathrm{H}, J=1.2,7.2 \mathrm{~Hz}), 7.46-7.24(\mathrm{~m}, 8 \mathrm{H}), 7.18-6.79(\mathrm{~m}, 13 \mathrm{H}), 6.68(\mathrm{dd}$, $1 \mathrm{H}, J=1.6,8.0 \mathrm{~Hz}), 4.88(\mathrm{~s}, 2 \mathrm{H}), 3.56(\mathrm{~s}, 3 \mathrm{H}), 2.73(\mathrm{t}, 2 \mathrm{H}, J=7.6 \mathrm{~Hz}), 2.58(\mathrm{t}, 2 \mathrm{H}, J=7.6 \mathrm{~Hz})$. Anal. Calcd for $\mathrm{C}_{40} \mathrm{H}_{32} \mathrm{~N}_{6} \mathrm{O}_{2} \mathrm{ClBr}(\%)$ : C: 64.57; H: 4.33; N: 11.29. Found (\%): C: 64.68; H: 4.40; N: 11.23 .

Methyl 5-iodo-1-[[2'-[[N-(2-chlorotrityl)]-1H-tetrazol-5-yl]biphenyl-4-yl]methyl]imidazole-4propanoate (17c). General procedure 3 was employed for the preparation of 17c using NIS in DMF. Yield 70\%; $\mathrm{R}_{f}=0.46$ (EtOAc); ESI-MS $(\mathrm{m} / \mathrm{z}): 792.48\left(\mathrm{M}+\mathrm{H}^{+}\right), 514.44\left(\mathrm{M}+\mathrm{H}^{+}-\mathrm{ClTr}\right), 277.76(\mathrm{ClTr})$; ${ }^{1} \mathrm{H}-\mathrm{NMR}\left(\mathrm{CD}_{3} \mathrm{OD}\right): \delta 7.58(\mathrm{~d}, 1 \mathrm{H}, J=7.6 \mathrm{~Hz}), 7.49-7.23(\mathrm{~m}, 13 \mathrm{H}), 7.16-7.00(\mathrm{~m}, 8 \mathrm{H}), 6.83(\mathrm{~d}, 1 \mathrm{H}$, $J=8.0 \mathrm{~Hz}), 5.15(\mathrm{~s}, 2 \mathrm{H}), 3.57(\mathrm{~s}, 3 \mathrm{H}), 2.78(\mathrm{t}, 2 \mathrm{H}, J=7.6 \mathrm{~Hz}), 2.57(\mathrm{t}, 2 \mathrm{H}, J=7.6 \mathrm{~Hz})$. Anal. Calcd for $\mathrm{C}_{40} \mathrm{H}_{32} \mathrm{~N}_{6} \mathrm{O}_{2} \mathrm{ClI}(\%)$ : C: 60.73; H: 4.08; N: 10.62. Found (\%): C: 60.66; H: 4.14; N: 10.56 .

(E)-Methyl 1-[[2'-(1H-tetrazol-5-yl)biphenyl-4-yl]methyl]imidazole-4-acrylate (18). General procedure 6 was employed for the preparation of 18. Yield 94\%; $\mathrm{R}_{f}=0.51\left(8.5: 1.5 \mathrm{CHCl}_{3}: \mathrm{MeOH}\right)$; ESI-MS $(\mathrm{m} / z)$ : $387.15\left(\mathrm{M}+\mathrm{H}^{+}\right)$; ${ }^{1} \mathrm{H}$ NMR (400 MHz, DMSO-d $)$ ): $\delta 8.49(\mathrm{~s}, 1 \mathrm{H}), 7.87(\mathrm{~s}, 1 \mathrm{H}), 7.68-7.54(\mathrm{~m}, 4 \mathrm{H}), 7.53$ $(\mathrm{d}, 1 \mathrm{H}, J=15.6 \mathrm{~Hz}), 7.27(\mathrm{~d}, 2 \mathrm{H}, J=7.6 \mathrm{~Hz}), 7.13(\mathrm{~d}, 2 \mathrm{H}, J=8.0 \mathrm{~Hz}), 6.48(\mathrm{~d}, 1 \mathrm{H}, J=15.6 \mathrm{~Hz}), 5.32$ (s, 2H), 3.71 (s, 3H). Anal. Calcd for $\mathrm{C}_{21} \mathrm{H}_{18} \mathrm{~N}_{6} \mathrm{O}_{2} \cdot \mathrm{CF}_{3} \mathrm{COOH}(\%)$ : C: 55.20; H: 3.83; N: 16.79. Found (\%): C: $55.11 ; \mathrm{H}: 3.72 ; \mathrm{N}: 16.84$.

Methyl 1-[[2'-(1H-tetrazol-5-yl)biphenyl-4-yl]methyl]imidazole-4-propanoate (19). General procedure 6 was employed for the preparation of 19. Yield 89\%; $\mathrm{R}_{f}=0.49\left(8.5: 1.5 \mathrm{CHCl}_{3}: \mathrm{MeOH}\right)$; ESI-MS $(\mathrm{m} / \mathrm{z}): 389.13\left(\mathrm{M}+\mathrm{H}^{+}\right) ;{ }^{1} \mathrm{H}-\mathrm{NMR}\left(\mathrm{CD}_{3} \mathrm{OD}\right): \delta 7.91(\mathrm{~d}, 1 \mathrm{H}, J=7.5 \mathrm{~Hz}), 7.62-6.76(\mathrm{~m}, 9 \mathrm{H}), 6.62(\mathrm{~s}, 1 \mathrm{H})$, $5.04(\mathrm{~s}, 2 \mathrm{H}), 3.64(\mathrm{~s}, 3 \mathrm{H}), 2.76(\mathrm{t}, 2 \mathrm{H}, J=7.2 \mathrm{~Hz}), 2.56(\mathrm{t}, 2 \mathrm{H}, J=7.2 \mathrm{~Hz}) ;{ }^{13} \mathrm{C}-\mathrm{NMR}\left(\mathrm{CD}_{3} \mathrm{OD}\right): \delta$ $167.99,163.90,141.79,141.32,140.68,139.38,138.96,137.53,136.06,135.08,131.47,130.72$, $130.30,129.66,128.36,127.80,127.31,126.52,114.82,50.85,50.37,35.75,29.83$. Anal. Calcd for $\mathrm{C}_{21} \mathrm{H}_{20} \mathrm{~N}_{6} \mathrm{O}_{2} \cdot \mathrm{CF}_{3} \mathrm{COOH}(\%)$ : C: 54.98; H: 4.21; N: 16.73. Found (\%): C: 54.86; H: 4.33; N: 16.61.

Methyl 5-chloro-1-[[2'-(1H-tetrazol-5-yl)biphenyl-4-yl] methyl]imidazole-4-propanoate (20a). General procedure 6 was employed for the preparation of 20a. Yield 95\%; $\mathrm{R}_{f}=0.53\left(8.5: 1.5 \mathrm{CHCl}_{3}: \mathrm{MeOH}\right)$; ESI-MS $(\mathrm{m} / \mathrm{z}): 423.92\left(\mathrm{M}+\mathrm{H}^{+}\right) ;{ }^{1} \mathrm{H}-\mathrm{NMR}\left(\mathrm{CD}_{3} \mathrm{OD}\right): \delta 8.30(\mathrm{~s}, 1 \mathrm{H}), 7.71-7.60(\mathrm{~m}, 2 \mathrm{H}), 7.23-7.18(\mathrm{~m}$, $4 \mathrm{H}), 5.32(\mathrm{~s}, 2 \mathrm{H}), 3.66(\mathrm{~s}, 3 \mathrm{H}), 2.92(\mathrm{t}, 2 \mathrm{H}, J=7.2 \mathrm{~Hz}), 2.70(\mathrm{t}, 2 \mathrm{H}, J=7.2 \mathrm{~Hz})$. Anal. Calcd for $\mathrm{C}_{21} \mathrm{H}_{19} \mathrm{~N}_{6} \mathrm{O}_{2} \mathrm{Cl} \cdot \mathrm{CF}_{3} \mathrm{COOH}(\%)$ : C: 51.45; H: 3.75; N: 15.65. Found (\%): C: 51.37; H: 3.83; N: 16.51.

Methyl 5-bromo-1-[[2'-(1H-tetrazol-5-yl)biphenyl-4-yl]methyl]imidazole-4-propanoate (20b). General procedure 6 was employed for the preparation of 20b. Yield 93\%; $\mathrm{R}_{f}=0.52\left(8.5: 1.5 \mathrm{CHCl}_{3}: \mathrm{MeOH}\right)$; ESI-MS $(\mathrm{m} / \mathrm{z}): 468.39\left(\mathrm{M}+\mathrm{H}^{+}\right)$; ${ }^{1} \mathrm{H}-\mathrm{NMR}\left(\mathrm{CD}_{3} \mathrm{OD}\right): \delta 8.80(\mathrm{~s}, 1 \mathrm{H}), 7.70-7.56(\mathrm{~m}, 4 \mathrm{H}), 7.26-7.18(\mathrm{~m}$, $4 \mathrm{H}), 5.40(\mathrm{~s}, 2 \mathrm{H}), 3.66(\mathrm{~s}, 3 \mathrm{H}), 2.97(\mathrm{t}, 2 \mathrm{H}, J=7.2 \mathrm{~Hz}), 2.73(\mathrm{t}, 2 \mathrm{H}, J=7.2 \mathrm{~Hz})$. Anal. Calcd for $\mathrm{C}_{21} \mathrm{H}_{19} \mathrm{~N}_{6} \mathrm{O}_{2} \mathrm{Br} \cdot \mathrm{CF}_{3} \mathrm{COOH}(\%)$ : C: 47.52; H: 3.47; N: 14.46. Found (\%): C: 47.63; H: 3.36; N: 14.55.

Methyl 5-iodo-1-[[2'-(1H-tetrazol-5-yl)biphenyl-4-yl]methyl]imidazole-4-propanoate (20c). General procedure 6 was employed for the preparation of 20c. Yield 95\%; $\mathrm{R}_{f}=0.53\left(8.5: 1.5 \mathrm{CHCl}_{3}: \mathrm{MeOH}\right)$; ESI-MS $(\mathrm{m} / \mathrm{z}): 515.38\left(\mathrm{M}+\mathrm{H}^{+}\right) ;{ }^{1} \mathrm{H}-\mathrm{NMR}\left(\mathrm{CD}_{3} \mathrm{OD}\right): \delta 8.37(\mathrm{~s}, 1 \mathrm{H}), 7.62-7.51(\mathrm{~m}, 4 \mathrm{H}), 7.09(\mathrm{~s}, 4 \mathrm{H})$, 
$5.23(\mathrm{~s}, 2 \mathrm{H}), 3.58(\mathrm{~s}, 3 \mathrm{H}), 2.84(\mathrm{t}, 2 \mathrm{H}, J=7.2 \mathrm{~Hz}), 2.60(\mathrm{t}, 2 \mathrm{H}, J=7.2 \mathrm{~Hz})$. Anal. Calcd for $\mathrm{C}_{21} \mathrm{H}_{19} \mathrm{~N}_{6} \mathrm{O}_{2} \mathrm{I} \cdot \mathrm{CF}_{3} \mathrm{COOH}(\%): \mathrm{C}: 43.96 ; \mathrm{H}: 3.21 ; \mathrm{N}: 13.37$. Found (\%): C: 43.87; H: 3.34; N: 13.44.

(E)-1-[[2'-[[N-(2-Chlorotrityl)]-1H-tetrazol-5-yl]biphenyl-4-yl]methyl]imidazole-4-acrylic acid (21a). General procedure 4 was employed for the preparation of 21a. Yield $90 \% ; \mathrm{R}_{f}=0.38(9: 1$ $\left.\mathrm{CHCl}_{3}: \mathrm{MeOH}\right)$; ESI-MS $(\mathrm{m} / \mathrm{z}): 650.17\left(\mathrm{M}+\mathrm{H}^{+}\right), 372.80\left(\mathrm{M}+\mathrm{H}^{+}-\mathrm{ClTr}\right), 277.76$ (ClTr); ${ }^{1} \mathrm{H}-\mathrm{NMR}$ (DMSO- $\left.d_{6}\right): \delta 7.81(\mathrm{~d}, 1 \mathrm{H}, J=7.6 \mathrm{~Hz}), 7.62-7.49(\mathrm{~m}, 4 \mathrm{H}), 7.45-7.30(\mathrm{~m}, 9 \mathrm{H}), 7.29(\mathrm{~d}, 1 \mathrm{H}, J=15.6 \mathrm{~Hz})$, $7.11-7.06(\mathrm{~m}, 4 \mathrm{H}), 6.78$ (d, 4H, $J=7.6 \mathrm{~Hz}), 6.68(\mathrm{~d}, 1 \mathrm{H}, J=8.0 \mathrm{~Hz}), 6.44$ (d, 1H, $J=15.6 \mathrm{~Hz}), 5.12$ (s, 2H). Anal. Calcd for $\mathrm{C}_{39} \mathrm{H}_{29} \mathrm{~N}_{6} \mathrm{O}_{2} \mathrm{Cl}(\%)$ : C: 72.16; H: 4.50; N: 12.95. Found (\%): C: 72.09; H: $4.58 ; \mathrm{N}: 12.89$.

(E)-(5-Methyl-2-oxo-1,3-dioxol)methyl-1-[[2'-[[N-(2-chlorotrityl)]-1H-tetrazol-5-yl]biphenyl-4-yl]methyl]imidazole-4-acrylate (21b). General procedure 5 was employed for the preparation of $21 \mathbf{b}$. Yield 84\%; $\mathrm{R}_{f}=0.52\left(\right.$ EtOAc); ESI-MS $(\mathrm{m} / \mathrm{z}): 762.165\left(\mathrm{M}+\mathrm{H}^{+}\right), 484.44\left(\mathrm{M}+\mathrm{H}^{+}-\mathrm{ClTr}\right), 277.76(\mathrm{ClTr})$; ${ }^{1} \mathrm{H}-\mathrm{NMR}\left(\mathrm{DMSO}-d_{6}\right): \delta 7.80(\mathrm{~s}, 2 \mathrm{H}), 7.60-7.42(\mathrm{~m}, 7 \mathrm{H}), 7.34-7.27(\mathrm{~m}, 7 \mathrm{H}), 7.09(\mathrm{~s}, 4 \mathrm{H}), 6.76(\mathrm{~s}, 4 \mathrm{H})$, $6.67(\mathrm{~s}, 1 \mathrm{H}), 6.28(\mathrm{~d}, 1 \mathrm{H}, J=16.0 \mathrm{~Hz}), 5.12(\mathrm{~s}, 2 \mathrm{H}), 5.02(\mathrm{~s}, 2 \mathrm{H}), 2.16(\mathrm{~s}, 3 \mathrm{H})$. Anal. Calcd for $\mathrm{C}_{44} \mathrm{H}_{33} \mathrm{~N}_{6} \mathrm{O}_{5} \mathrm{Cl}(\%)$ : C: 69.42; H: 4.37; N: 11.04. Found (\%): C: 69.39; H: 4.32; N: 10.98.

(E)-1-[[2'-(1H-Tetrazol-5-yl)biphenyl-4-yl]methyl]imidazole-4-acrylic acid (22a). General procedure 6 was employed for the preparation of 22a. Yield 95\%; $\mathrm{R}_{f}=0.38\left(4: 1: 1 n\right.$-butanol:acetic acid: $\left.\mathrm{H}_{2} \mathrm{O}\right)$; ESI-MS $(m / z): 373.44\left(\mathrm{M}+\mathrm{H}^{+}\right) ;{ }^{1} \mathrm{H}-\mathrm{NMR}\left(\mathrm{DMSO}-d_{6}\right): \delta 8.56(\mathrm{~s}, 1 \mathrm{H}), 7.84(\mathrm{~s}, 1 \mathrm{H}), 7.66(\mathrm{~s}, 2 \mathrm{H}), 7.58$ $(\mathrm{s}, 1 \mathrm{H}), 7.52(\mathrm{~s}, 1 \mathrm{H}), 7.42(\mathrm{~d}, 1 \mathrm{H}, J=16.0 \mathrm{~Hz}), 7.26(\mathrm{~s}, 2 \mathrm{H}), 7.11(\mathrm{~s}, 2 \mathrm{H}), 6.40(\mathrm{~d}, 1 \mathrm{H}, J=16.0 \mathrm{~Hz})$, 5.31 (s, 2H). Anal. Calcd for $\mathrm{C}_{20} \mathrm{H}_{16} \mathrm{~N}_{6} \mathrm{O}_{2} \cdot \mathrm{CF}_{3} \mathrm{COOH}(\%)$ : C: 54.32; $\mathrm{H}: 3.52 ; \mathrm{N}: 17.28$. Found (\%): C: 54.45; H: $3.41 ; \mathrm{N}: 17.39$.

(E)-(5-Methyl-2-oxo-1,3-dioxol)methyl-1-[[2'-(1H-tetrazol-5-yl)biphenyl-4-yl]methyl]imidazole-4-acrylate (22b). General procedure 6 was employed for the preparation of 22b. Yield 94\%; $\mathrm{R}_{f}=0.52(8.5: 1.5$ $\left.\mathrm{CHCl}_{3}: \mathrm{MeOH}\right)$; ESI-MS $(\mathrm{m} / \mathrm{z}): 485.52\left(\mathrm{M}+\mathrm{H}^{+}\right)$; ${ }^{1} \mathrm{H}-\mathrm{NMR}\left(\mathrm{DMSO}-d_{6}\right): \delta 8.10(\mathrm{~s}, 1 \mathrm{H}), 7.76(\mathrm{~s}, 1 \mathrm{H})$, $7.65(\mathrm{~s}, 2 \mathrm{H}), 7.57-7.51(\mathrm{~m}, 3 \mathrm{H}), 7.24-7.20(\mathrm{~m}, 2 \mathrm{H}), 7.11-7.07(\mathrm{~m}, 2 \mathrm{H}), 6.38(\mathrm{~d}, 1 \mathrm{H}, J=16.0 \mathrm{~Hz}), 5.24$ (s, 2H), $5.04(\mathrm{~s}, 2 \mathrm{H}), 2.16(\mathrm{~s}, 3 \mathrm{H})$. Anal. Calcd for $\mathrm{C}_{25} \mathrm{H}_{20} \mathrm{~N}_{6} \mathrm{O}_{5} \cdot \mathrm{CF}_{3} \mathrm{COOH}(\%): \mathrm{C}: 54.18 ; \mathrm{H}: 3.54$; N: 14.04. Found (\%): C: 54.08; H: 3.41; N: 14.17.

1-[[2'-[[N-(2-Chlorotrityl)]-1H-tetrazol-5-yl]biphenyl-4-yl]methyl]imidazole-4-propanoic acid (23a). General procedure 4 was employed for the preparation of 23a. Yield 93\%; $\mathrm{R}_{f}=0.33(9: 1$ $\left.\mathrm{CHCl}_{3}: \mathrm{MeOH}\right)$; ESI-MS $(\mathrm{m} / \mathrm{z}): 652.19\left(\mathrm{M}+\mathrm{H}^{+}\right), 374.77\left(\mathrm{M}+\mathrm{H}^{+}-\mathrm{ClTr}\right), 277.77$ (ClTr); ${ }^{1} \mathrm{H}-\mathrm{NMR}$ $\left(\mathrm{DMSO}-d_{6}\right): \delta 7.82-7.69(\mathrm{~m}, 1 \mathrm{H}), 7.64-7.44(\mathrm{~m}, 6 \mathrm{H}), 7.40-7.27(\mathrm{~m}, 7 \mathrm{H}), 7.10-7.02(\mathrm{~m}, 4 \mathrm{H})$, $7.09-6.66(\mathrm{~m}, 6 \mathrm{H}), 5.00(\mathrm{~s}, 2 \mathrm{H}), 2.60(\mathrm{t}, 2 \mathrm{H}, J=7.2 \mathrm{~Hz}), 2.38(\mathrm{t}, 2 \mathrm{H}, J=7.2 \mathrm{~Hz})$. Anal. Calcd for $\mathrm{C}_{39} \mathrm{H}_{31} \mathrm{~N}_{6} \mathrm{O}_{2} \mathrm{Cl}(\%)$ : C: 71.94; H: 4.80; N: 12.91. Found (\%): C: 71.87; H: 4.86; N: 12.87.

(5-Methyl-2-oxo-1,3-dioxol)methyl-1-[[2'-[[N-(2-chlorotrityl)]-1H-tetrazol-5-yl]biphenyl-4-yl]-methyl] imidazole-4-propanoate (23b). General procedure 5 was employed for the preparation of $\mathbf{2 3 b}$. Yield 73\%; $\mathrm{R}_{f}=0.45\left(9: 1 \mathrm{CHCl}_{3}: \mathrm{MeOH}\right) ; \operatorname{ESI}-\mathrm{MS}(\mathrm{m} / \mathrm{z}): 764.21\left(\mathrm{M}+\mathrm{H}^{+}\right), 486.56\left(\mathrm{M}+\mathrm{H}^{+}-\mathrm{ClTr}\right), 277.76$ $(\mathrm{ClTr}) ;{ }^{1} \mathrm{H}-\mathrm{NMR}\left(\mathrm{CDCl}_{3}\right): \delta 7.96(\mathrm{~d}, 1 \mathrm{H}, J=7.2 \mathrm{~Hz}), 7.49-7.31(\mathrm{~m}, 9 \mathrm{H}), 7.22-7.18(\mathrm{~m}, 5 \mathrm{H}), 7.13(\mathrm{~d}$, 
$2 \mathrm{H}, J=8.0 \mathrm{~Hz}), 6.92-6.84(\mathrm{~m}, 5 \mathrm{H}), 6.82(\mathrm{~s}, 1 \mathrm{H}), 6.72(\mathrm{~d}, 1 \mathrm{H}, J=8.0 \mathrm{~Hz}), 4.88(\mathrm{~s}, 2 \mathrm{H}), 4.81(\mathrm{~s}, 2 \mathrm{H})$, $2.83(\mathrm{t}, 2 \mathrm{H}, J=7.2 \mathrm{~Hz}), 2.68(\mathrm{t}, 2 \mathrm{H}, J=7.2 \mathrm{~Hz}), 2.16(\mathrm{~s}, 3 \mathrm{H})$. Anal. Calcd for $\mathrm{C}_{44} \mathrm{H}_{35} \mathrm{~N}_{6} \mathrm{O}_{5} \mathrm{Cl}(\%)$ : C: 69.24; H: 4.62; N: 11.01. Found (\%): C: 69.19; H: 4.67; N: 11.08.

1-[[2'-(1H-Tetrazol-5-yl)biphenyl-4-yl]methyl]imidazole-4-propanoic acid (24a). General procedure 6 was employed for the preparation of 24a. Yield 92\%; $\mathrm{R}_{f}=0.33\left(4: 1: 1 n\right.$-butanol:acetic acid: $\left.\mathrm{H}_{2} \mathrm{O}\right)$; ESI-MS $(\mathrm{m} / \mathrm{z}): 375.41\left(\mathrm{M}+\mathrm{H}^{+}\right) ;{ }^{1} \mathrm{H}-\mathrm{NMR}\left(\mathrm{CD}_{3} \mathrm{OD}\right): \delta 8.89(\mathrm{~s}, 1 \mathrm{H}), 7.71-7.67(\mathrm{~m}, 2 \mathrm{H}), 7.60-7.56$ (m, 2H), $7.39(\mathrm{~s}, 1 \mathrm{H}), 7.35(\mathrm{~d}, 2 \mathrm{H}, J=7.2 \mathrm{~Hz}), 7.20(\mathrm{~d}, 2 \mathrm{H}, J=7.2 \mathrm{~Hz}), 5.50(\mathrm{~s}, 2 \mathrm{H}), 2.98(\mathrm{t}, 2 \mathrm{H}$ $J=7.2 \mathrm{~Hz}$ ), 2.71 (t, $2 \mathrm{H}, J=7.2 \mathrm{~Hz}$ ). Anal. Calcd for $\mathrm{C}_{20} \mathrm{H}_{18} \mathrm{~N}_{6} \mathrm{O}_{2} \cdot \mathrm{CF}_{3} \mathrm{COOH}(\%): \mathrm{C}: 54.10 ; \mathrm{H}: 3.92$; $\mathrm{N}: 17.21$. Found (\%): C: 54.17; H: 3.81; N: 17.32.

(5-Methyl-2-oxo-1,3-dioxol)methyl-1-[[2'-(1H-tetrazol-5-yl)biphenyl-4-yl]methyl]imidazole-4-propanoate (24b). General procedure 6 was employed for the preparation of 24b. Yield 87\%; $\mathrm{R}_{f}=0.49(8.5: 1.5$ $\left.\mathrm{CHCl}_{3}: \mathrm{MeOH}\right) ; \quad$ ESI-MS $(\mathrm{m} / \mathrm{z}): 487.56 \quad\left(\mathrm{M}+\mathrm{H}^{+}\right) ;{ }^{1} \mathrm{H}-\mathrm{NMR} \quad\left(\mathrm{DMSO}-d_{6}\right): \quad \delta \quad 9.06 \quad(\mathrm{~s}, \quad 1 \mathrm{H})$, 7.67-7.12 (m, 9H), 5.35 (s, 2H), 4.94 (s, 2H), 2.87 (t, 2H, J=7.2 Hz), 2.74 (t, 2H, J=7.2 Hz), 2.12 (s, $3 \mathrm{H})$. Anal. Calcd for $\mathrm{C}_{25} \mathrm{H}_{22} \mathrm{~N}_{6} \mathrm{O}_{5} \cdot \mathrm{CF}_{3} \mathrm{COOH}(\%)$ : C: 54.00; H: 3.86; N: 13.99. Found (\%): C: 53.91; $\mathrm{H}: 3.91 ; \mathrm{N}: 14.11$.

5-Chloro-1-[[2'-[[N-(2-chlorotrityl)]-1H-tetrazol-5-yl]biphenyl-4-yl] methyl]imidazole-4-propanoic acid (25a). General procedure 4 was employed for the preparation of 25a. Yield 94\%; $\mathrm{R}_{f}=0.39$ (9:1 $\left.\mathrm{CHCl}_{3}: \mathrm{MeOH}\right)$; ESI-MS $(\mathrm{m} / \mathrm{z}): 686.66\left(\mathrm{M}+\mathrm{H}^{+}\right), 408.92\left(\mathrm{M}+\mathrm{H}^{+}-\mathrm{ClTr}\right), 277.81$ (ClTr); ${ }^{1} \mathrm{H}-\mathrm{NMR}$ $\left(\mathrm{CDCl}_{3}\right): \delta 7.98-7.94(\mathrm{~m}, 1 \mathrm{H}), 7.48-7.08(\mathrm{~m}, 15 \mathrm{H}), 6.85(\mathrm{~d}, 6 \mathrm{H}, J=7.6 \mathrm{~Hz}), 6.71(\mathrm{~d}, 1 \mathrm{H}, J=8.0 \mathrm{~Hz})$, 4.84 (s, 2H), 2.79 (bs, 2H), 2.58 (bs, $2 \mathrm{H})$. Anal. Calcd for $\mathrm{C}_{39} \mathrm{H}_{30} \mathrm{~N}_{6} \mathrm{O}_{2} \mathrm{Cl}_{2}(\%): \mathrm{C}: 68.31 ; \mathrm{H}: 4.41$; N: 12.26. Found (\%): C: $68.08 ; \mathrm{H}: 4.53 ; \mathrm{N}: 12.57$.

(5-Methyl-2-oxo-1,3-dioxol)methyl-5-chloro-1-[[2'-[[N-(2-chlorotrityl)]-1H-tetrazol-5-yl]biphenyl-4yl]methyl]imidazole-4-propanoate (25b). General procedure 1 was employed for the preparation of 7. Yield 82\%; $\mathrm{R}_{f}=0.50$ (EtOAc); ESI-MS $(\mathrm{m} / \mathrm{z}): 798.72\left(\mathrm{M}+\mathrm{H}^{+}\right), 521.03\left(\mathrm{M}+\mathrm{H}^{+}-\mathrm{ClTr}\right), 277.78(\mathrm{ClTr})$; ${ }^{1} \mathrm{H}-\mathrm{NMR}\left(\mathrm{CDCl}_{3}\right): \delta 7.95(\mathrm{~d}, 1 \mathrm{H}, J=7.2 \mathrm{~Hz}), 7.48-7.34(\mathrm{~m}, 6 \mathrm{H}), 7.34-7.32(\mathrm{~m}, 5 \mathrm{H}), 7.23(\mathrm{~m}, 5 \mathrm{H})$, $7.11(\mathrm{~d}, 2 \mathrm{H}, J=8.0 \mathrm{~Hz}), 6.91-6.86(\mathrm{~m}, 8 \mathrm{H}), 4.93(\mathrm{~s}, 2 \mathrm{H}), 4.83(\mathrm{~s}, 2 \mathrm{H}), 2.87(\mathrm{t}, 2 \mathrm{H}, J=7.2 \mathrm{~Hz}), 2.74(\mathrm{t}$, $2 \mathrm{H}, J=7.2 \mathrm{~Hz}), 2.15$ (s, 3H). Anal. Calcd for $\mathrm{C}_{44} \mathrm{H}_{34} \mathrm{~N}_{6} \mathrm{O}_{5} \mathrm{Cl}_{2}(\%)$ : C: 66.25; H: 4.30; N: 10.54. Found (\%): C: $66.24 ; \mathrm{H}: 4.18 ; \mathrm{N}: 10.33$.

5-Bromo-1-[[2'-[[N-(2-chlorotrityl)]-1H-tetrazol-5-yl]biphenyl-4-yl] methyl]imidazole-4-propanoic acid (25c). General procedure 4 was employed for the preparation of 25c. Yield 91\%; $\mathrm{R}_{f}=0.37$ (9:1, $\left.\mathrm{CHCl}_{3}: \mathrm{MeOH}\right)$; ESI-MS $(\mathrm{m} / \mathrm{z})$ : $731.11\left(\mathrm{M}+\mathrm{H}^{+}\right), 453.36\left(\mathrm{M}+\mathrm{H}^{+}-\mathrm{ClTr}\right), 277.76$ (ClTr); ${ }^{1} \mathrm{H}-\mathrm{NMR}$ $\left(\mathrm{CDCl}_{3}\right): \delta 8.04(\mathrm{~d}, 1 \mathrm{H}, J=7.6 \mathrm{~Hz}), 7.67-7.28(\mathrm{~m}, 15 \mathrm{H}), 7.09(\mathrm{~d}, 2 \mathrm{H}, J=8.0 \mathrm{~Hz}), 6.97(\mathrm{~d}, 4 \mathrm{H}, J=7.6$ $\mathrm{Hz}), 6.87(\mathrm{~d}, 1 \mathrm{H}, J=8.0 \mathrm{~Hz}), 5.12(\mathrm{~s}, 1 \mathrm{H}), 2.96-2.92(\mathrm{~m}, 2 \mathrm{H}), 2.81-2.78(\mathrm{~m}, 2 \mathrm{H})$. Anal. Calcd for $\mathrm{C}_{39} \mathrm{H}_{30} \mathrm{~N}_{6} \mathrm{O}_{2} \mathrm{ClBr}(\%): \mathrm{C}: 64.16 ; \mathrm{H}: 4.14 ; \mathrm{N}: 11.51$. Found (\%): C: 64.11; H: 4.21; N: 11.44.

(5-Methyl-2-oxo-1,3-dioxol)methyl-5-bromo-1-[[2'-[[N-(2-chlorotrityl)]-1H-tetrazol-5-yl]biphenyl-4yl]methyl]imidazole-4-propanoate (25d). General procedure 5 was employed for the preparation of 25d. Yield 72\%; $\mathrm{R}_{f}=0.48$ (EtOAc); ESI-MS $(\mathrm{m} / \mathrm{z}): 843.18\left(\mathrm{M}+\mathrm{H}^{+}\right), 565.43\left(\mathrm{M}+\mathrm{H}^{+}-\mathrm{ClTr}\right), 277.78$ 
(ClTr); ${ }^{1} \mathrm{H}-\mathrm{NMR}\left(\mathrm{CDCl}_{3}\right): \delta 8.03(\mathrm{~d}, 1 \mathrm{H}, J=7.2 \mathrm{~Hz}), 7.65-7.33(\mathrm{~m}, 13 \mathrm{H}), 7.24(\mathrm{~d}, 2 \mathrm{H}, J=7.6 \mathrm{~Hz})$, $6.99(\mathrm{~m}, 4 \mathrm{H}), 6.87(\mathrm{~d}, 1 \mathrm{H}, J=7.6 \mathrm{~Hz}), 5.07(\mathrm{~s}, 2 \mathrm{H}), 4.93(\mathrm{~s}, 2 \mathrm{H}), 2.94(\mathrm{t}, 2 \mathrm{H}, J=6.4 \mathrm{~Hz}), 2.82(\mathrm{t}, 2 \mathrm{H}$, $J=6.4 \mathrm{~Hz}$ ), $2.24(\mathrm{~s}, 3 \mathrm{H})$. Anal. Calcd for $\mathrm{C}_{44} \mathrm{H}_{34} \mathrm{~N}_{6} \mathrm{O}_{5} \mathrm{ClBr}(\%)$ : C: 62.75; H: 4.07; N: 9.98. Found (\%): C: 62.68; H: 4.15; N: 9.91.

5-Iodo-1-[[2'-[[N-(2-chlorotrityl)]-1H-tetrazol-5-yl]biphenyl-4-yl] methyl]imidazole-4-propanoic acid (25e). General procedure 4 was employed for the preparation of 25e. Yield 95\%; $\mathrm{R}_{f}=0.37(9: 1$ $\left.\mathrm{CHCl}_{3}: \mathrm{MeOH}\right)$; ESI-MS $(\mathrm{m} / \mathrm{z})$ : $778.13\left(\mathrm{M}+\mathrm{H}^{+}\right), 500.35\left(\mathrm{M}+\mathrm{H}^{+}-\mathrm{ClTr}\right), 277.76$ (ClTr); ${ }^{1} \mathrm{H}-\mathrm{NMR}$ $\left(\mathrm{CDCl}_{3}\right): \delta 7.97(\mathrm{~d}, 1 \mathrm{H}, J=7.6 \mathrm{~Hz}), 7.72(\mathrm{~s}, 1 \mathrm{H}), 7.49-7.17(\mathrm{~m}, 15 \mathrm{H}), 6.93(\mathrm{~d}, 2 \mathrm{H}, J=7.6 \mathrm{~Hz}), 6.88$ $(\mathrm{d}, 4 \mathrm{H}, J=7.6 \mathrm{~Hz}), 6.73(\mathrm{~d}, 1 \mathrm{H}, J=7.2 \mathrm{~Hz}), 4.98(\mathrm{~s}, 2 \mathrm{H}), 2.88(\mathrm{t}, 2 \mathrm{H}, J=7.2 \mathrm{~Hz}), 2.73(\mathrm{t}, 2 \mathrm{H}$, $J=7.2 \mathrm{~Hz}$ ). Anal. Calcd for $\mathrm{C}_{39} \mathrm{H}_{30} \mathrm{~N}_{6} \mathrm{O}_{2} \mathrm{ClI}(\%)$ : C: 60.28; H: 3.89; N: 10.82. Found (\%): C: 60.21; H: $3.96 ; \mathrm{N}: 10.73$.

(5-Methyl-2-oxo-1,3-dioxol)methyl-5-iodo-1-[[2'-[[N-(2-chlorotrityl)]-1H-tetrazol-5-yl]biphenyl-4yl]methyl]imidazole-4-propanoate (25f). General procedure 5 was employed for the preparation of 25f. Yield 74\%; $\mathrm{R}_{f}=0.51$ (EtOAc); ESI-MS $(\mathrm{m} / \mathrm{z}): 890.21\left(\mathrm{M}+\mathrm{H}^{+}\right), 612.45\left(\mathrm{M}+\mathrm{H}^{+}-\mathrm{ClTr}\right), 276.88$ (ClTr); ${ }^{1} \mathrm{H}-\mathrm{NMR}\left(\mathrm{CDCl}_{3}\right): \delta 7.77(\mathrm{~d}, 1 \mathrm{H}, J=7.6 \mathrm{~Hz}), 7.45-7.22(\mathrm{~m}, 8 \mathrm{H}), 7.15-6.80(\mathrm{~m}, 14 \mathrm{H}), 4.86(\mathrm{~s}$, $2 \mathrm{H}), 4.71(\mathrm{~s}, 2 \mathrm{H}), 2.71(\mathrm{t}, 2 \mathrm{H}, J=7.2 \mathrm{~Hz}), 2.59(\mathrm{t}, 2 \mathrm{H}, J=7.2 \mathrm{~Hz}), 2.02(\mathrm{~s}, 3 \mathrm{H})$; Anal. Calcd for $\mathrm{C}_{44} \mathrm{H}_{34} \mathrm{~N}_{6} \mathrm{O}_{5} \mathrm{ClI}(\%)$ : C: 59.44; H: 3.85; N: 9.45. Found (\%): C: 59.36; H: 3.94; N: 4.37.

5-Chloro-1-[[2'-(1H-tetrazol-5-yl)biphenyl-4-yl]methyl]imidazole-4-propanoic acid (26a). General procedure 6 was employed for the preparation of 26a. Yield 90\%; $\mathrm{R}_{f}=0.32$ (4:1:1 $n$-butanol:acetic acid: $\left.\mathrm{H}_{2} \mathrm{O}\right)$; ESI-MS $(\mathrm{m} / \mathrm{z}): 409.31\left(\mathrm{M}+\mathrm{H}^{+}\right) ;{ }^{1} \mathrm{H}-\mathrm{NMR}\left(\mathrm{CD}_{3} \mathrm{OD}\right): \delta 8.62(\mathrm{~s}, 1 \mathrm{H}), 7.70(\mathrm{~d}, 2 \mathrm{H}, J=7.2$ $\mathrm{Hz}), 7.62-7.56(\mathrm{~m}, 2 \mathrm{H}), 7.27(\mathrm{~d}, 2 \mathrm{H}, J=7.6 \mathrm{~Hz}), 7.19$ (d, 2H, $J=7.6 \mathrm{~Hz}), 5.38(\mathrm{~s}, 2 \mathrm{H}), 2.94(\mathrm{t}, 2 \mathrm{H}$, $J=7.2 \mathrm{~Hz}$ ), 2.69 (t, $2 \mathrm{H}, J=7.2 \mathrm{~Hz}$ ). Anal. Calcd for $\mathrm{C}_{20} \mathrm{H}_{17} \mathrm{~N}_{6} \mathrm{O}_{2} \mathrm{Cl} \cdot \mathrm{CF}_{3} \mathrm{COOH}(\%): \mathrm{C}: 50.54 ; \mathrm{H}: 3.47$; $\mathrm{N}: 16.07$. Found (\%): C: $50.43 ; \mathrm{H}: 3.58 ; \mathrm{N}: 16.17$.

(5-Methyl-2-oxo-1,3-dioxol)methyl-5-chloro-1-[[2'-(1H-tetrazol-5-yl)biphenyl-4-yl]methyl]imidazole4-propanoate (26b). General procedure 6 was employed for the preparation of $\mathbf{2 6 b}$. Yield 89\%; $\mathrm{R}_{f}=0.56\left(8.5: 1.5 \mathrm{CHCl}_{3}: \mathrm{MeOH}\right)$; ESI-MS $(\mathrm{m} / \mathrm{z}): 522.00\left(\mathrm{M}+\mathrm{H}^{+}\right) ;{ }^{1} \mathrm{H}-\mathrm{NMR}\left(\mathrm{DMSO}-d_{6}\right): \delta 8.24(\mathrm{~s}$, 1H), 7.71-7.54 (m, 4H), 7.17-7.08 (m, 4H), $5.24(\mathrm{~s}, 2 \mathrm{H}), 4.92(\mathrm{~s}, 2 \mathrm{H}), 2.76(\mathrm{t}, 2 \mathrm{H}, J=7.6 \mathrm{~Hz}), 2.69$ (t, $2 \mathrm{H}, J=7.6 \mathrm{~Hz}$ ), 2.13 (s, 3H). Anal. Calcd for $\mathrm{C}_{25} \mathrm{H}_{21} \mathrm{~N}_{6} \mathrm{O}_{5} \mathrm{Cl} \cdot \mathrm{CF}_{3} \mathrm{COOH}(\%): \mathrm{C}: 51.07$; H: 3.49; N: 13.24. Found (\%): C: $51.13 ; \mathrm{H}: 3.41 ; \mathrm{N}: 13.35$.

5-Bromo-1-[[2'-(1H-tetrazol-5-yl)biphenyl-4-yl]methyl]imidazole-4-propanoic acid (26c). General procedure 6 was employed for the preparation of 26c. Yield 93\%; $\mathrm{R}_{f}=0.31$ (4:1:1 $n$-butanol:acetic acid: $\left.\mathrm{H}_{2} \mathrm{O}\right)$; ESI-MS $(\mathrm{m} / \mathrm{z}): 454.37\left(\mathrm{M}+\mathrm{H}^{+}\right) ;{ }^{1} \mathrm{H}-\mathrm{NMR}\left(\mathrm{CD}_{3} \mathrm{OD}\right): \delta 7.67-7.55(\mathrm{~m}, 5 \mathrm{H}), 7.12(\mathrm{~s}, 4 \mathrm{H})$, $5.21(\mathrm{~s}, 2 \mathrm{H}), 2.83(\mathrm{t}, 2 \mathrm{H}, J=7.2 \mathrm{~Hz}), 2.60(\mathrm{t}, 2 \mathrm{H}, J=7.2 \mathrm{~Hz})$. Anal. Calcd for $\mathrm{C}_{20} \mathrm{H}_{17} \mathrm{~N}_{6} \mathrm{O}_{2} \mathrm{Br} \cdot \mathrm{CF}_{3} \mathrm{COOH}(\%)$ : C: 46.58; H: 3.20; N: 14.81. Found (\%): C: 46.63; H: 3.32; N: 14.69.

(5-Methyl-2-oxo-1,3-dioxol)methyl-5-bromo-1-[[2'-(1H-tetrazol-5-yl)biphenyl-4-yl]methyl]imidazole4-propanoate (26d). General procedure 6 was employed for the preparation of 26d. Yield 91\%; $\mathrm{R}_{f}=0.55\left(8.5: 1.5 \mathrm{CHCl}_{3}: \mathrm{MeOH}\right)$; ESI-MS $(\mathrm{m} / \mathrm{z}): 566.45\left(\mathrm{M}+\mathrm{H}^{+}\right) ;{ }^{1} \mathrm{H}-\mathrm{NMR}\left(\mathrm{DMSO}-d_{6}\right): \delta 7.91(\mathrm{~s}$, 
1H), 7.67-7.64 (m, 2H), 7.58-7.53 (m, 2H), $7.09(\mathrm{~s}, 4 \mathrm{H}), 5.17(\mathrm{~s}, 2 \mathrm{H}), 4.92(\mathrm{~s}, 2 \mathrm{H}), 2.72-2.63(\mathrm{~m}$, $4 \mathrm{H}), 2.13(\mathrm{~s}, 3 \mathrm{H})$. Anal. Calcd for $\mathrm{C}_{25} \mathrm{H}_{21} \mathrm{~N}_{6} \mathrm{O}_{5} \mathrm{Br} \cdot \mathrm{CF}_{3} \mathrm{COOH}(\%)$ : C: 47.73; H: 3.26; N: 12.37. Found (\%): C: 47.83; H: 3.34; N: 13.27 .

5-Iodo-1-[[2'-(1H-tetrazol-5-yl)biphenyl-4-yl]methyl]imidazole-4-propanoic acid (26e). General procedure 6 was employed for the preparation of 26e. Yield 91\%, $\mathrm{R}_{f}=0.29$ (4:1:1 $n$-butanol:acetic acid: $\left.\mathrm{H}_{2} \mathrm{O}\right)$; ESI-MS $(\mathrm{m} / \mathrm{z}): 501.38\left(\mathrm{M}+\mathrm{H}^{+}\right) ;{ }^{1} \mathrm{H}-\mathrm{NMR}\left(\mathrm{CD}_{3} \mathrm{OD}\right): \delta 8.79(\mathrm{~s}, 1 \mathrm{H}), 7.68-7.64(\mathrm{~m}, 2 \mathrm{H})$, 7.56-7.31 (m, 4H), $7.19(\mathrm{~d}, 2 \mathrm{H}, J=8.0 \mathrm{~Hz}), 5.35(\mathrm{~s}, 2 \mathrm{H}), 2.95(\mathrm{t}, 2 \mathrm{H}, J=7.2 \mathrm{~Hz}), 2.68(\mathrm{t}, 2 \mathrm{H}$, $J=7.2 \mathrm{~Hz}$ ). Anal. Calcd for $\mathrm{C}_{20} \mathrm{H}_{17} \mathrm{~N}_{6} \mathrm{O}_{2} \mathrm{I} \cdot \mathrm{CF}_{3} \mathrm{COOH}(\%)$ : C: 43.01; H: 2.95; N: 13.68. Found (\%): C: 43.13; H: 2.87; N: 13.79 .

(5-Methyl-2-oxo-1,3-dioxol)methyl-5-iodo-1-[[2'-(1H-tetrazol-5-yl)biphenyl-4-yl]methyl]imidazole-4propanoate (26f). General procedure 6 was employed for the preparation of 26f. Yield 90\%; $\mathrm{R}_{f}=0.56$ (8.5:1.5 $\left.\mathrm{CHCl}_{3}: \mathrm{MeOH}\right)$; ESI-MS $(\mathrm{m} / \mathrm{z}): 613.44\left(\mathrm{M}+\mathrm{H}^{+}\right) ;{ }^{1} \mathrm{H}-\mathrm{NMR}\left(\mathrm{CD}_{3} \mathrm{OD}\right): \delta 8.00(\mathrm{~s}, 1 \mathrm{H}), 7.66-7.56$ $(\mathrm{m}, 4 \mathrm{H}), 7.15(\mathrm{bs}, 4 \mathrm{H}), 5.22(\mathrm{~s}, 2 \mathrm{H}), 4.88(\mathrm{~s}, 2 \mathrm{H}), 2.86(\mathrm{t}, 2 \mathrm{H}, J=7.2 \mathrm{~Hz}), 2.70(\mathrm{t}, 2 \mathrm{H}$, $J=7.2 \mathrm{~Hz}), 2.16(\mathrm{~s}, 3 \mathrm{H})$. Anal. Calcd for $\mathrm{C}_{25} \mathrm{H}_{21} \mathrm{~N}_{6} \mathrm{O}_{5} \mathrm{I} \cdot \mathrm{CF}_{3} \mathrm{COOH}(\%)$ : C: 44.64; H: 3.05; N: 11.57. Found (\%): C: 44.72; H: 3.14; N: 11.65.

\subsubsection{Synthesis of 1,5-Disubstituted Benzyl Analogues 30, 32}

(E)-Methyl 3-[1-[(2-(trimethylsilyl)ethoxy)methyl]-1H-imidazole-4-yl]acrylate (27). To a solution of $(E)$-urocanic methyl ester (6) $(0.61 \mathrm{~g}, 4.03 \mathrm{mmol})$ in dry DMF $(15.0 \mathrm{~mL})$ under argon atmosphere at $0{ }^{\circ} \mathrm{C}$ was added dry $\mathrm{NaH}$ (powdered $95 \%, 0.12 \mathrm{~g}, 4.84 \mathrm{mmol}$ ) and the suspension was left at the same temperature for $15 \mathrm{~min}$. Then, SEM-Cl $(0.68 \mathrm{~mL}, 4.84 \mathrm{mmol})$ was added in three portions and the reaction mixture was allowed to warm to $\mathrm{rt}$ for $4 \mathrm{~h}$. The reaction was quenched with $0.5 \mathrm{~N} \mathrm{NaOH}$ $(15 \mathrm{~mL})$ and extracted with $\mathrm{CH}_{2} \mathrm{Cl}_{2}(3 \times 20 \mathrm{~mL})$. The combined organic phases were washed with brine $(\times 2)$, dried $\left(\mathrm{Na}_{2} \mathrm{SO}_{4}\right)$ and concentrated in vacuo. Purification by flash column chromatography (7:3 EtOAc:hexanes) afforded 27. Yield 78\%; $\mathrm{R}_{f}=0.35$ (8:2 EtOAc:hexanes); ESI-MS ( $\left.\mathrm{m} / \mathrm{z}\right): 283.29$ $\left(\mathrm{M}+\mathrm{H}^{+}\right) ;{ }^{1} \mathrm{H}-\mathrm{NMR}\left(\mathrm{CDCl}_{3}\right): \delta 8.58(\mathrm{~s}, 1 \mathrm{H}), 7.52(\mathrm{~d}, 1 \mathrm{H}, J=16.0 \mathrm{~Hz}), 7.35(\mathrm{~s}, 1 \mathrm{H}), 6.68(\mathrm{~d}, 1 \mathrm{H}$, $J=16.0 \mathrm{~Hz}), 5.42(\mathrm{~s}, 2 \mathrm{H}), 3.82(\mathrm{~s}, 3 \mathrm{H}), 3.58(\mathrm{t}, 2 \mathrm{H}, J=8.0 \mathrm{~Hz}), 0.96(\mathrm{t}, 2 \mathrm{H}, J=8.0 \mathrm{~Hz}), 0.01(\mathrm{~s}, 9 \mathrm{H})$;

${ }^{13} \mathrm{C}-\mathrm{NMR}\left(\mathrm{CDCl}_{3}\right): \delta$ 166.64, 137.98, 134.48, 130.47, 121.21, 120.59, 50.85, 76.79, 68.14, 51.95, 18.22, 1.44. Anal. Calcd for $\mathrm{C}_{13} \mathrm{H}_{22} \mathrm{~N}_{2} \mathrm{O}_{3} \mathrm{Si}$ (\%): C: 55.29; H: 7.85; N:9.92. Found (\%): C:55.36; $\mathrm{H}: 7.72 ; \mathrm{N}: 9.87$.

Methyl 3-[1-[(2-(trimethylsilyl)ethoxy)methyl]-1H-imidazole-4-yl]propanoate (28). General procedure 2 was employed for the preparation of 28. Yield 91\%; $\mathrm{R}_{f}=0.44\left(9: 1 \mathrm{CHCl}_{3}: \mathrm{MeOH}\right)$; ESI-MS $(\mathrm{m} / z)$ : $284.99\left(\mathrm{M}+\mathrm{H}^{+}\right) ;{ }^{1} \mathrm{H}-\mathrm{NMR}\left(\mathrm{CDCl}_{3}\right): \delta 7.91(\mathrm{~s}, 1 \mathrm{H}), 6.88(\mathrm{~s}, 1 \mathrm{H}), 5.25(\mathrm{~s}, 2 \mathrm{H}), 3.67(\mathrm{~s}, 3 \mathrm{H}), 3.50(\mathrm{t}, 2 \mathrm{H}$, $J=8.0 \mathrm{~Hz}), 3.00(\mathrm{t}, 2 \mathrm{H}, J=7.2 \mathrm{~Hz}), 2.72(\mathrm{t}, 2 \mathrm{H}, J=7.2 \mathrm{~Hz}), 0.91(\mathrm{t}, 2 \mathrm{H}, J=8.0 \mathrm{~Hz}), 0.01(\mathrm{~s}, 9 \mathrm{H})$;

${ }^{13} \mathrm{C}-\mathrm{NMR}\left(\mathrm{CDCl}_{3}\right): \delta 173.13,136.14,116.19,76.88,67.08,120.59,51.65,33.27,22.27,17.89,1.44$. Anal. Calcd for $\mathrm{C}_{13} \mathrm{H}_{24} \mathrm{~N}_{2} \mathrm{O}_{3} \mathrm{Si}(\%)$ : C:54.90; H:8.51; N:9.85. Found (\%): C:54.81; H:8.61; N:9.74.

Methyl 1-[[2'-[[N-(2-chlorotrityl)]-1H-tetrazol-5-yl]biphenyl-4-yl]methyl]-3-[(2-(trimethylsilyl)ethoxy)methyl]imidazolium-5-propanoate bromide (29). To a stirred solution of 28 (2.0 g, $7.03 \mathrm{mmol})$ 
in dry $\mathrm{CH}_{2} \mathrm{Cl}_{2}(20.0 \mathrm{~mL})$ under argon was added the alkylating agent $4(4.31 \mathrm{~g}, 7.73 \mathrm{mmol})$ in one portion and the resulting mixture was heated under reflux for $3 \mathrm{~h}$. Upon completion (disappearance of starting material confirmed by RP-HPLC), the solvent was concentrated, followed by chromatographic purification $\left(97: 3 \mathrm{CHCl}_{3}: \mathrm{MeOH}\right)$ to afford 29 as a white powder. Yield $81 \% ; \mathrm{R}_{f}=0.40(9: 1$ $\left.\mathrm{CHCl}_{3}: \mathrm{MeOH}\right)$; ESI-MS (m/z): $796.19\left(\mathrm{M}^{+}-\mathrm{Br}\right) ;{ }^{1} \mathrm{H}-\mathrm{NMR}\left(\mathrm{CDCl}_{3}\right): \delta 7.93(\mathrm{dd}, 1 \mathrm{H}, J=1.6,7.6 \mathrm{~Hz})$, $7.52-7.46(\mathrm{~m}, 3 \mathrm{H}), 7.40-7.13(\mathrm{~m}, 13 \mathrm{H}), 7.11(\mathrm{~s}, 1 \mathrm{H}), 6.94(\mathrm{~d}, 6 \mathrm{H}, J=7.6 \mathrm{~Hz}), 5.67(\mathrm{~s}, 2 \mathrm{H}), 5.46$ (s, 2H), 3.75-3.64 (m, 5H), $2.75(\mathrm{t}, 2 \mathrm{H}, J=7.2 \mathrm{~Hz}), 2.47$ (t, 2H, $J=7.2 \mathrm{~Hz}), 0.94$ (t, 2H, $J=8.0 \mathrm{~Hz}$ ), 0.002 (s, 9H). Anal. Calcd for $\mathrm{C}_{46} \mathrm{H}_{48} \mathrm{~N}_{6} \mathrm{O}_{3} \mathrm{SiBr}(\%)$ : C:63.04; H:5.52; N:9.59. Found (\%): C:62.93; $\mathrm{H}: 5.57 ; \mathrm{N}: 9.51$.

Methyl 1-[[2'-(1H-tetrazol-5-yl)biphenyl-4-yl]methyl]-3-[(2-(trimethylsilyl)ethoxy)methyl]imidazole5-propanoate (30). General procedure 6 was employed for the preparation of $\mathbf{3 0}$. Yield $90 \% ; \mathrm{R}_{f}=0.53$ (8.5:1.5 $\left.\mathrm{CHCl}_{3}: \mathrm{MeOH}\right)$; ESI-MS $(m / z): 389.51\left(\mathrm{M}+\mathrm{H}^{+}\right) ;{ }^{1} \mathrm{H}-\mathrm{NMR}\left(\mathrm{DMSO}-d_{6}\right): \delta 9.13(\mathrm{~s}, 1 \mathrm{H}), 7.68(\mathrm{~d}$, $1 \mathrm{H}, J=8.0 \mathrm{~Hz}), 7.59(\mathrm{~d}, 1 \mathrm{H}, J=7.6 \mathrm{~Hz}), 7.54(\mathrm{~d}, 1 \mathrm{H}, J=7.6 \mathrm{~Hz}), 7.50(\mathrm{~s}, 1 \mathrm{H}), 7.21(\mathrm{~d}, 2 \mathrm{H}, J=8.0 \mathrm{~Hz})$, $7.14(\mathrm{~d}, 1 \mathrm{H}, J=8.0 \mathrm{~Hz}), 5.48(\mathrm{~s}, 2 \mathrm{H}), 3.60(\mathrm{~s}, 3 \mathrm{H}), 2.77$ (t, 2H, $J=7.2 \mathrm{~Hz}), 2.64$ (t, 2H, $J=7.2 \mathrm{~Hz})$. Anal. Calcd for $\mathrm{C}_{21} \mathrm{H}_{20} \mathrm{~N}_{6} \mathrm{O}_{2} \cdot \mathrm{CF}_{3} \mathrm{COOH}(\%)$ : C:54.98; H:4.21; N:16.73. Found (\%): C:54.91; H:4.13; N:16.61.

Methyl 2-hydroxymethyl-1-[[2'-[[N-(2-chlorotrityl)]-1H-tetrazol-5-yl]biphenyl-4-yl]methyl]-3-[(2(trimethylsilyl)ethoxy)methyl]imidazolium-5-propanoate bromide (31). In a sealed tube were sequentially added 29 (2.0 g, $2.38 \mathrm{mmol})$, DMF (1.0 mL), 37\% formalin (2.65 mL, $35.63 \mathrm{mmol})$ and diisopropylethylamine $(2.02 \mathrm{~mL}, 11.90 \mathrm{mmol})$. The resulting mixure was stirred at $85{ }^{\circ} \mathrm{C}$ until HPLC showed no starting material left ( $c a .1 \mathrm{~h}$ ). Then, the mixture was quenched with $5 \%$ aqueous citric acid $(10 \mathrm{~mL})$, extracted with $\mathrm{CH}_{2} \mathrm{Cl}_{2}$ and the combined organic phases were washed with brine, dried $\left(\mathrm{Na}_{2} \mathrm{SO}_{4}\right)$, filtered and concentrated in vacuo. Purification by flash column chromatography (96:4 $\left.\mathrm{CHCl}_{3}: \mathrm{MeOH}\right)$ afforded 31. Yield 91\%; $\mathrm{R}_{f}=0.28\left(9: 1 \mathrm{CHCl}_{3}: \mathrm{MeOH}\right)$; ESI-MS $(m / z): 826.32\left(\mathrm{M}^{+}-\mathrm{Br}\right)$; ${ }^{1} \mathrm{H}-\mathrm{NMR}\left(\mathrm{CDCl}_{3}\right): \delta 7.95(\mathrm{~d}, 1 \mathrm{H}, J=6.8 \mathrm{~Hz}), 7.52-7.42(\mathrm{~m}, 3 \mathrm{H}), 7.40-7.26(\mathrm{~m}, 9 \mathrm{H}),, 7.19(\mathrm{~s}, 1 \mathrm{H})$, $7.15(\mathrm{~d}, 2 \mathrm{H}, J=8.0 \mathrm{~Hz}), 6.94-6.89(\mathrm{~m}, 8 \mathrm{H}), 5.76(\mathrm{~s}, 2 \mathrm{H}), 5.52(\mathrm{~s}, 2 \mathrm{H}), 4.72(\mathrm{~s}, 2 \mathrm{H}), 3.70-3.62(\mathrm{~m}$, $5 \mathrm{H}), 2.76(\mathrm{t}, 2 \mathrm{H}, J=6.8 \mathrm{~Hz}), 2.54(\mathrm{t}, 2 \mathrm{H}, J=6.8 \mathrm{~Hz}), 0.95(\mathrm{t}, 2 \mathrm{H}, J=8.0 \mathrm{~Hz}), 0.001$ (s, 9H). Anal. Calcd for $\mathrm{C}_{47} \mathrm{H}_{50} \mathrm{~N}_{6} \mathrm{O}_{4} \mathrm{SiBr}(\%)$ : C:62.28; H:5.56; N:9.27. Found (\%): C:62.37; H:5.43; N:9.19.

Methyl 2-hydroxymethyl-1-[[2'-(1H-tetrazol-5-yl)biphenyl-4-yl]methyl]-3-[(2-(trimethylsilyl)ethoxy)methyl]imidazole-5-propanoate (32). General procedure 6 was employed for the preparation of 32. Yield 88\%; $\mathrm{R}_{f}=0.32\left(8: 2 \mathrm{CHCl}_{3}: \mathrm{MeOH}\right)$; ESI-MS $(\mathrm{m} / \mathrm{z}): 419.56\left(\mathrm{M}+\mathrm{H}^{+}\right) ;{ }^{1} \mathrm{H}-\mathrm{NMR}\left(\mathrm{DMSO}-d_{6}\right): \delta$ $7.67(\mathrm{dd}, 2 \mathrm{H}, J=2.0,7.6 \mathrm{~Hz}), 7.59$ (d, 1H, $J=7.6 \mathrm{~Hz}), 7.52$ (d, 1H, J=7.6 Hz), $7.44(\mathrm{~s}, 1 \mathrm{H}), 7.12$ (s, $4 \mathrm{H}), 6.26(\mathrm{bs}, 1 \mathrm{H}), 5.45(\mathrm{~s}, 2 \mathrm{H}), 4.74(\mathrm{~s}, 2 \mathrm{H}), 3.58(\mathrm{~s}, 3 \mathrm{H}), 2.71(\mathrm{t}, 2 \mathrm{H}, J=6.8 \mathrm{~Hz}), 2.61(\mathrm{t}, 2 \mathrm{H}, J=6.8$ $\mathrm{Hz}$ ). Anal. Calcd for $\mathrm{C}_{22} \mathrm{H}_{22} \mathrm{~N}_{6} \mathrm{O}_{3} \cdot \mathrm{CF}_{3} \mathrm{COOH}(\%)$ : C:54.14; H:4.35; N:15.78. Found (\%): C:54.23; $\mathrm{H}: 4.25 ; \mathrm{N}: 15.86$.

\subsection{Pharmacological Evaluation}

\subsubsection{Radioligand Binding Assay}

Radioligand binding assay was performed as previously described [20]. 


\subsection{Docking Studies}

The 3D model of the AT1 receptor used in our docking studies was kindly provided by Tuccinardi et al. [29]. The construction of this model is based on X-ray bovine rhodopsin structure, molecular procedure and available site-directed mutagenesis data [30]. Molecular Docking studies were performed using Glide extra precision (XP) implemented Induced Fit Docking (IFD) protocol (v 5.0) [31-33] docking programs under the Linux operating system. The active site was defined by $20 \AA$ inner cubic grid box, centered on the point that is the center of mass of residues Lys199 and His256. The IFD protocol under the Schrodinger molecular modeling package was used in order to eliminate clashes between receptor and ligand atoms and for the receptor to gain partial flexibility to the receptor. Before the docking simulations, the complexes were submitted to the protein preparation module of Schrodinger. Ligands were constructed using the Schrodinger's Maestro module and then geometry optimization was performed for these ligands using Polak-Ribiere conjugate gradient (PRCG) minimization $\left(0.0001 \mathrm{~kJ}^{-1} \mathrm{~mol}^{-1}\right.$ convergence criteria). Protonation states of residues were created using LigPrep and Protein Preparation modules under the Schrodinger package at neutral $\mathrm{pH}$. IFD uses the Glide docking program to account the ligand flexibility and the refinement module and the Prime (v.1.6) program [32,33] to account for flexibility of the receptor. Schrodinger's IFD protocol model uses the following steps (the description below is taken from the IFD user manual): (i) Constrained minimization of the receptor with an RMSD cutoff of $0.18 \AA$. (ii) Initial Glide docking of each ligand using soft potentials ( 0.5 van der Waals radii scaling of non-polar atoms of ligands and receptor using partial charge cutoff of 0.15). (iii) Derived docking poses were refined using the Prime Induced Fit module of Schrodinger. Residues within $5.0 \AA$ of ligand poses were minimized in order to form suitable conformations of poses at the active site of the receptor. (iv) Glide re-docking of each protein-ligand complex.

\section{Conclusions}

In the present study, we have demonstrated an efficient and convenient strategy for the syntheses of a series of $N$-benzyl and $N$-biphenylmethyl imidazole derivatives substituted either at the $N-1$ or $N-3$ positions of $(E)$-urocanic acid. A facile and clean methodology with a few-step synthetic protocol in high yields has been developed. Biological evaluation of the synthesized analogues concerning their binding affinity for the AT1 receptor revealed that certain analogues (compounds 12 and 18) are moderate inhibitors. In particular, the methyl acrylate analogue $\mathbf{1 8}$ which bears the biphenylmethyl moiety, showed relevant higher activity compared to the others. In addition, the lack of a lipophilic alkyl chain may also explain the lower activity of $\mathbf{1 8}$ which seems to be critical for binding affinity, compared to losartan. Docking results showed that flexibility of these molecules is an important factor that governs their drug activity. The synthesized analogues adopt different orientations in the active site as indicated by the docking studies. It is reasonable to assume that the studied molecules first adopt the most comfortable conformation and orientation and show the highest scoring when these are approaching the receptor. Such a hypothesis can explain the experimental data which have indicated the poor activity of the studied molecules. The propensity of some molecules to adopt the appropriate orientation and thus exert all favored but not maximal interactions can explain their moderate activity. 


\section{Acknowledgments}

This research project was supported by the research programmes PRAXE B-EPAN \& 4NEW_B_2012, General Secretariat of Research and Technology of Greece, Ministry of Development and the pharmaceutical companies ELDRUG SA and VIANEX SA. We also acknowledge Demetrios Vahliotis (Instrumental Analysis Laboratory, University of Patras) for recording the NMR spectra. C. Koukoulitsa acknowledges Greek national funds through the Operational Program "Education and Lifelong Learning" of the National Strategic Reference Framework (NSRF)-Research Funding Program: Heracleitus II, investing in knowledge society through the European Social Fund.

\section{Conflict of Interest}

The authors have no financial/commercial conflicts of interest to declare.

\section{References}

1. Naik, P.; Murumkar, P.; Giridhar, R.; Yadav, M.R. Angiotensin II receptor type $1\left(\mathrm{AT}_{1}\right)$ selective nonpeptidic antagonists-A perspective. Bioorg. Med. Chem. 2010, 18, 8418-8456.

2. Burnier, M. Angiotensin II Type 1 Receptor Blockers. Circulation 2001, 103, 904-912.

3. Burnier, M.; Brunner, H.R. Angiotensin II receptor antagonists. Lancet 2000, 355, 637-645.

4. Cappelli, A.; Mohr, G.P.; Gallelli, A.; Rizzo, M.; Anzini, M.; Vomero, S.; Mennuni, L.; Ferrari, F.; Makovec, F.; Menziani, M.C.; et al. Design, synthesis, structural studies, biological evaluation, and computational simulations of novel potent AT1 angiotensin II receptor antagonists based on the 4-phenylquinoline structure. J. Med. Chem. 2004, 47, 2574-2586.

5. Cappelli, A.; Nannicini, C.; Giuliani, G.; Valenti, S.; Mohr, G.P.; Anzini, M.; Mennuni, L.; Ferrari, F.; Caselli, G.; Giordani, A.; et al. Design, synthesis, and biological evaluation of AT1 angiotensin II receptor antagonists based on the pyrazolo[3,4-b]pyridine and related heteroaromatic bicyclic systems. J. Med. Chem. 2008, 51, 2137-2146.

6. Kubo, K.; Kohara, Y.; Imamiya, E.; Sugiura, Y.; Inada, Y.; Furukawa, Y.; Nishikawa, K.; Naka, T. Nonpeptide angiotensin II receptor antagonists. Synthesis and biological activity of benzimidazolecarboxylic acids. J. Med. Chem. 1993, 36, 2182-2195.

7. Duncia, J.V.; Chiu, A.T.; Carini, D.J.; Gregory, G.B.; Johnson, A.L.; Price, W.A.; Wells, G.J.; Wong, P.C.; Calabrese, J.C.; Timmermans, P.B.M.W.M. The discovery of potent nonpeptide angiotensin II receptor antagonists: A new class of potent antihypertensives. J. Med. Chem. 1990, 33, 1312-1329.

8. Carini, D.J.; Duncia, J.V.; Aldrich, P.E.; Chiu, A.T.; Johnson, A.L.; Pierce, M.E.; Price, W.A.; Santella, J.B.; Wells, G.J.; Wexler, R.R.; et al. Nonpeptide angiotensin II receptor antagonists: The discovery of a series of $\mathrm{N}$-(biphenylylmethyl)imidazoles as potent, orally active antihypertensives. J. Med. Chem. 1991, 34, 2525-2547.

9. Yanagisawa, H.; Ameniya, Y.; Kanazaki, T.; Shimoji, Y.; Fujimoto, K.; Kitahara, Y.M.; Sada, T.; Mizuno, M.; Ikeda, M.; Miyamoto, S.; et al. Nonpeptide angiotensin II receptor antagonists: Synthesis, biological activities, and structure-activity relationships of imidazole-5-carboxylic acids bearing alkyl, alkenyl, and hydroxyalkyl substituents at the 4-position and their related compounds. J. Med. Chem. 1996, 39, 323-338. 
10. Kohara, Y.; Kubo, K.; Imamiya, E.; Wada, T.; Inada, Y.; Naka T. Synthesis and angiotensin II receptor antagonistic activities of benzimidazole derivatives bearing acidic heterocycles as novel tetrazole bioisosteres. J. Med. Chem. 1996, 39, 5228-5235.

11. Baker, W.L.; White, W.B. Azilsartan medoxomil: A new angiotensin II receptor antagonist for treatment of hypertension. Ann. Pharmacother. 2011, 45, 1506-1515.

12. Miura, S.; Karnik, S.S.; Saku, K. Significance of pigment spithelium-derived factor levels with angiotensin II type 1 receptor blokers in patients with successful colonary stent implantation. J. Renin. Angiotensin. Aldosterone. Syst. 2011, 12, 1-7.

13. Herr, R.J. 5-Substituted-1H-tetrazoles as carboxylic acid isosteres: Medicinal chemistry and synthetic methods. Bioorg. Med. Chem. 2002, 10, 3379-3393.

14. Deprez, P.; Guillaume, J.; Becker, R.; Corbier, A.; Didierlaurent, S.; Fortin, M.; Frechet, D.; Hamon, G.; Heckmann, B.; Heitch, H.; et al. Sulfonylureas and sulfonylcarbamates as new non-tetrazole angiotensin II receptor antagonists. Discovery of a highly potent orally active (Imidazolylbiphenylyl)sulfonylurea (HR 720). J. Med. Chem. 1995, 38, 2357-2377.

15. Ismail, M.A.H.; Barker, S.; Abou El Ella, D.A.; Abouzid, K.A.M.; Toubar, R.A.; Todd, M.H. Design and synthesis of new tetrazolyl- and carboxy-biphenylylmethyl-quinazolin-4-one derivatives as angiotensin II AT1 Receptor Antagonists. J. Med. Chem. 2006, 49, 1526-1535.

16. Xu, J.Y.; Zeng, Y.; Ran, Q.; Wie, Z.; Bi, Y.; He, Q.H.; Wang, Q.J.; Hu, S.; Zhang, J.; Tang, M.Y.; et al. Synthesis and biological activity of 2-alkylbenzimidazoles bearing a $N$-phenylpyrrole moiety as novel angiotensin II AT1 receptor antagonists. Bioorg. Med. Chem. Lett. 2007, 17, 2921-2926.

17. Kaur, N.; Kaur, A.; Bansal, Y.; Shah, D.V.; Bansal, G.; Singh, M. Design, synthesis, and evaluation of 5-sulfamoyl benzimidazole derivatives as novel angiotensin II receptor antagonists. Bioorg. Med. Chem. 2008, 16, 10210-10215.

18. Agelis, G.; Resvani, A.; Durdagi, S.; Spyridaki, K.; Tumova, T.; Slaninova, J.; Giannopoulos, P.; Vlahakos, D.; Liapakis, G.; Mavromoustakos, T.; et al. The discovery of new potent non-peptide angiotensin II AT1 receptor blockers: A concise synthesis, molecular docking studies and biological evaluation of $\mathrm{N}$-substituted 5-butylimidazole derivatives. Eur. J. Med. Chem. 2012, 55, 358-374.

19. Agelis, G.; Roumelioti, P.; Resvani, A.; Durdagi, S.; Androutsou, M.E.; Kelaidonis, K.; Mavromoustakos, T.; Matsoukas, J. An efficient synthesis of a rationally designed 1,5 disubstituted imidazole AT1 angiotensin II receptor antagonist: Reorientation of imidazole pharmacophore groups in losartan reserves high receptor affinity and confirms docking studies. J. Comput.-Aided. Mol. Des. 2010, 24, 749-758.

20. Agelis, G.; Resvani, A.; Matsoukas, M.T.; Tselios, T.; Kelaidonis, K.; Kalavrizioti, D.; Vlahakos, D.; Matsoukas, J. Towards non-peptide ANG II AT1 receptor antagonists based on urocanic acid: rational design, synthesis and biological evaluation. Amino Acids 2011, 40, 411-420.

21. Weinstock, J.; Keenan, R.M.; Samanen, J.; Hempel, J.; Finkelstein, J.A.; Franz, R.G.; Gaitanopoulos, D.E.; Girard, G.R.; Gleason, J.G.; Hill, D.T.; et al. 1-(Carboxybenzyl)imidazole5-acrylic acids: Potent and selective angiotensin II receptor antagonists. J. Med. Chem. 1991, 34, 1514-1517. 
22. Keenan, R.M.; Weinstock, J.; Finkelstein, J.A.; Franz, R.G.; Gaitanopoulos, D.E.; Girard, G.R.; Hill, D.T.; Morgan, T.M.; Samanen, J.; Peishoff, C.E.; et al. Potent nonpeptide angiotensin II receptor antagonists. 2. 1-(Carboxybenzyl)imidazole-5-acrylic acids. J. Med. Chem. 1993, 36, 1880-1892.

23. Bovy, P.R.; Reitz, D.B.; Collins, J.T.; Chamberlain, T.S.; Olins, G.M.; Corpus, V.M.; McMahon, E.G.; Palomo, M.A.; Koepke, J.P.; Smits, G.J.; et al. Nonpeptide angiotensin II antagonists: $N$-phenyl-1H-pyrrole derivatives are angiotensin II receptor antagonists. J. Med. Chem. 1993, 36, 101-110.

24. Kristensen, J.; Lysén, M.; Begtrup, M. Synthesis of ortho substituted arylboronic esters by in situ trapping of unstable lithio intermediates. Org. Lett. 2001, 3, 1435-1437.

25. Amantini, D.; Beleggia, R.; Fringuelli, F.; Pizzo, F.; Vaccaro, L. TBAF-catalyzed synthesis of 5-substituted $1 H$-tetrazoles under solventless conditions. J. Org. Chem. 2004, 69, 2896-2898.

26. Pirrung, M.C.; Pei, T. Synthesis of (+/-)-homohistidine. J. Org. Chem. 2000, 65, 2229-2230.

27. Lipshutz, B.H.; Vaccaro, W.; Huff, B. Protection of imidazoles as their $\beta$-trimethylsilylethoxymethyl (SEM) derivatives. Tetrahedron Lett. 1986, 27, 4095-4098.

28. Luo, G.; Chen, L.; Dubowchik, G. Regioselective Protection at N-2 and Derivatization at $C-3$ of Indazoles. J. Org. Chem. 2006, 71, 5392-5395.

29. Tuccinardi, T.; Calderone, V.; Rapposelli, S.; Martinelli, A. Proposal of a new binding orientation for non-peptide AT1 antagonists: Homology modeling, docking and three-dimensional quantitative structure-activity relationship analysis. J. Med. Chem. 2006, 49, 4305-4316.

30. Okada, T.; Sugihara, M.; Bondar, A.N.; Elstner, M.; Entel, P.; Buss, V. The retinal conformation and its environment in rhodopsin in light of a new $2.2 \AA$ crystal structure. J. Mol. Biol. 2004, 342, 571-583.

31. Friesner, R.A.; Murphy, R.B.; Repasky, M.P.; Frye, L.L.; Greenwood, J.R.; Halgren, T.A.; Sanschagrin, P.C.; Mainz, D.T. Extra precision glide: Docking and scoring incorporating a model of hydrophobic enclosure for protein-ligand complexes. J. Med. Chem. 2006, 49, 6177-6196.

32. Sherman, W.; Day, T.; Jacobson, M.P.; Friesner, R.A.; Farid, R. Novel procedure for modeling ligand/receptor induced fit effects. J. Med. Chem. 2006, 49, 534-553.

33. Small-Molecule Drug Discovery Suite 2012: Glide, version 5.8; Schrödinger, LLC: New York, NY, USA, 2012.

Sample Availability: Samples of the final compounds 12, 18, 30, 32 are available from the authors.

(C) 2013 by the authors; licensee MDPI, Basel, Switzerland. This article is an open access article distributed under the terms and conditions of the Creative Commons Attribution license (http://creativecommons.org/licenses/by/3.0/). 